\title{
Light elements synthesized in the He-layer and the H-rich envelope of a type II supernova -Influence of the adopted neutrino emission model-
}

\author{
Takashi Yoshida, Hiroyuki Emori, and Kiyoshi Nakazawa \\ Department of Earth and Planetary Sciences, Tokyo Institute of Technology, Meguro, Tokyo 152-8551, Japan
}

(Received May 6, 1999; Revised October 6, 1999; Accepted December 16, 1999)

\begin{abstract}
In order to establish a suitable manner for finding presolar grains of supernova origin, we simulated the explosive nucleosynthesis of light elements, i.e., CNO-elements and $X$-elements ( $\mathrm{Li}, \mathrm{Be}$, and B), in the He-layer and the $\mathrm{H}$ rich envelope of a $16.2 M_{\odot}$ supernova and calculated their final abundances and abundance ratios using the nuclear reaction network. We also investigated the response of the synthesized abundances of light elements to the change of strength and duration of the neutrino emission, about which we have not a precise knowledge. The obtained results are as follows. The amounts of ${ }^{6} \mathrm{Li}$ and ${ }^{9} \mathrm{Be}$ produced during the supernova explosion are quite small. The ratios of ${ }^{6} \mathrm{Li} /{ }^{7} \mathrm{Li}$ and ${ }^{9} \mathrm{Be} /{ }^{7} \mathrm{Li}$ are less than $2 \times 10^{-4}$, which are much smaller than the corresponding solar-system values. The other $X$-elements and CNO-elements (except ${ }^{12} \mathrm{C}$ and ${ }^{16} \mathrm{O}$ ) are synthesized, more or less, and their abundances depend strongly on the internal mass coordinate as well as the adopted neutrino emission model. However, ${ }^{11} \mathrm{~B} /{ }^{7} \mathrm{Li}$ ratio and isotopic ratios of $\mathrm{CNO}$-elements are confined within one order of magnitude or so. On the basis of the above results, we finally proposed useful diagrams between two isotopic (elemental) ratios, which would help us to find presolar grains of supernova origin.
\end{abstract}

\section{Introduction}

In the last decade, remarkable progress of chemical/isotopic analyses (e.g., the Secondary Ion Mass Spectrometry, SIMS), have made it possible to determine precisely chemical/isotopic compositions in a very tiny area of a cosmochemical sample (e.g., Mckeegan et al., 1985; Hoppe et al., 1995). By the use of such superior tools, many authors have tried to determine chemical compositions of small components in meteorites (such as $\mu m$-size refractory grains) and found a lot of $\mu \mathrm{m}$-size grains the isotopic ratios of which are quite different from the solar-system composition. Such exotic grains are called presolar grains (Zinner, 1998). A large isotopic heterogeneity of presolar grains would be kept so far from their birth and would reflect physical and chemical birth conditions.

Evidence for presolar grains was firstly provided by large isotopic anomalies in carbon (Swart et al., 1983). After measurement of the chemical compositions of $\mu \mathrm{m}$-size isolated grains was established by the use of SIMS (Zinner et al., 1989), presolar grains were found in SiC (Zinner et al., 1989), graphite (Amari et al., 1990), corundum (Hutcheon et $a l ., 1994)$, and so on (reviewed in Zinner, 1998). By the discovery of presolar grains, our new interest is aroused: where were they born? how was the isotopic heterogeneity brought about? As the origin of presolar grains, mainly two possibilities are proposed; one is the supernova origin and another is the AGB (asymptotic giant branch) star origin.

Copy right (C) The Society of Geomagnetism and Earth, Planetary and Space Sciences (SGEPSS); The Seismological Society of Japan; The Volcanological Society of Japan; The Geodetic Society of Japan; The Japanese Society for Planetary Sciences.
Presolar grains originated from supernovae are identified by comparing between their isotopic ratios and those predicted by supernova nucleosynthesis theory. Most of the identifications have been conducted in Washington Univ. group (e.g., Zinner, 1998). Until now, some presolar grains have been identified as supernova origin by the use of their ${ }^{44} \mathrm{Ca}$ excess (Nittler et al., 1996). However, some of these grains also show silicon or nitrogen isotopic ratios outside the range of the ratios predicted by the supernova nucleosynthesis theory (Travaglio et al., 1999). Since these unsolved problems still remain, we can say nothing about their origin definitely. This is due, partly, to the facts that we have still a small number of sample grains and that isotopic species, which we can analyze precisely, are limited owing to the smallness of a sample particle. However, the most significant reason is that we have not an enough and a general knowledge on the nucleosynthesis in the supernova explosion. Thus, it is very important to investigate extensively the nucleosynthesis in the supernova explosion and to grasp a general behavior of isotopic variation of the supernova ejecta.

Since Arnett (1969), many efforts have been dedicated for studying the nucleosynthesis in supernovae on the basis of the stellar evolution of massive stars as well as the model of hydrodynamical explosion. Recently, Woosley and his collaborators investigated extensively element synthesis in supernovae; Woosley and Weaver (1995) obtained chemical compositions of supernova ejecta for a grid of stellar masses and metallicities, and also described synthetic processes extensively for most of isotopes. Meyer et al. (1995) tabulated the amounts of 103 isotopes till $\mathrm{Zn}$ synthesized in eight distinct zones of the ejecta of a $25 M_{\odot}$ supernova. 
Similarly, Nomoto and his collaborators studied gigantically the explosive nucleosynthesis in supernovae (e.g., Nomoto et al., 1984; Shigeyama et al., 1987; Thielemann et al., 1990; Thielemann et al., 1996; Iwamoto et al., 1997; Nakamura et al., 1999). Thielemann et al. (1990) investigated the chemical composition synthesized in SN1987A. In Thielemann et al. (1996), they explored the nucleosynthesis in supernovae with various masses paying their attention to the sensitivity of the amounts of synthesized isotopes to various parameters (such as the progenitor mass and the explosion energy).

However, such intensive studies are not so useful for us: their interest focused on the survey of the nucleosynthesis over almost all of isotopes and the reconstruction of global scenario on the formation of elements. Or, they aimed at the explanation of observational features of special supernova events. Thus, we should investigate explosive synthetic processes concentrating on some isotopes and viewing from a different angle, i.e., varieties in elemental ratios from an explosion rather than the total amounts of the elements in a whole star.

As readily conjectured and as shown in previous works, the explosive nucleosynthesis is affected intricately by various physical/chemical conditions. The amounts of elements synthesized during the supernova explosion may vary widely owing to the initial chemical composition, strength and duration of the neutrino flux, the mass of progenitor, and others. The synthesized amounts must be also influenced by whether there occurs a mixing (due to the Rayleigh-Taylor instability) between different layers in the supernova ejecta or not. Purposes of a series of our papers are to investigate the explosive nucleosynthesis under the various physical/chemical conditions mentioned above and to find characteristic behaviors of the abundances and the ratios of elements synthesized in the supernova explosion, which are useful for distinguishing presolar grains of supernova origin from others.

In the present paper and the subsequent papers, we have paid special attention to the explosive nucleosynthesis of light elements, i.e., CNO-elements and the light element $\mathrm{Li}, \mathrm{Be}$, and $\mathrm{B}$ (hereafter we call them the $X$-elements). Oxygen is one of the main constituents of silicate grains. Carbon and Nitrogen are generally abundant and a part of them, at least, would be extracted into dust grains when they condense in the cooling stage of supernova explosion. In the case of the $X$-elements, despite the low solar-system abundances, abundance values in the silicate bearing particles are relatively high because of high chemical affinity with $\mathrm{Si}-\mathrm{Mg}$ grains and their high refractory features. In this sense, we can expect that the grains of supernova origin would certainly contain these elements. In addition, the $X$-elements and the CNOelements behave in very different ways in the nucleosynthesis. Proposal of the criteria to identify the presolar grains in these two kinds of elements will give us good double check.

Several authors have proposed some pairs of isotopes to identify presolar grains from meteorites. If we could believe that the meteorites keep their original chemical properties, we would challenge to find the best way to identify the presolar grains. However, actually meteorites were exposed to cosmic rays for a long time and they had many chances of alternation in formation process of solar nebula such as chondrule formation. As long as we cannot be free from a concerns of these pollutions or contaminations to the meteorites, it is better to have as many criteria as possible in order to identify the presolar grains.

In a series of our studies we concentrate on the explosive nucleosynthesis only in the He-layer and the H-rich envelope. Previous studies on the explosive nucleosynthesis indicated that the $\mathrm{CNO}$-elements (except ${ }^{12} \mathrm{C}$ and ${ }^{16} \mathrm{O}$ ) as well as the $X$-elements are produced by appreciable amounts in these layers (Woosley et al., 1990; Woosley and Weaver, 1995; Meyer et al., 1995).

In this study we will investigate behavior of light elements, which are synthesized in the He-layer and the H-rich envelope of a $16.2 M_{\odot}$ supernova which corresponds to $20 M_{\odot}$ main sequence star and was reduced by $3.8 M_{\odot}$ of mass loss, and seek useful diagrams among the isotopic (elemental) ratios of light elements for selection of presolar grains of supernova origin taking account of the sensitivity to the adopted model of the neutrino emission (i.e., strength and duration of the emitted neutrino flux) as the first step to obtain general behavior of light elements synthesized in supernovae. On the $X$-elements, neutrino-induced reactions have important roles in their synthetic processes. Woosley and his collaborators focused on the neutrino-induced nucleosynthesis (Epstein et al., 1988) and Woosley et al. (1990) clarified firstly the explosive nucleosynthesis including the neutrino-induced nuclear processing in realistic stellar conditions. They investigated production processes of the $X$-elements and their sensitivity to the neutrino temperatures. However, as regards the influence of duration and strength of the emitted neutrino flux, they concentrated on that on neutron flux, so they did not investigate influence on the $X$-element synthesis. So, in order to investigate varieties of the amounts and the elemental ratios of the $X$-elements, we should also take account of the influence of the neutrino emission model such as duration and strength of the emitted neutrino flux.

We will introduce a specific term of "detection limit". Roughly speaking, dust grains are piles of oxygen atoms and, hence, the amounts of other species (except $\mathrm{Si}, \mathrm{Mg}$, and $\mathrm{Fe}$ ) are much less than that of oxygen. When dust grains contain some kind of rare elements with the content of $1 \times 10^{-7}$ times as small as that of ${ }^{16} \mathrm{O}$, it is difficult to detect such rare elements, even with the most sensitive instruments including ion probe technique. Expecting an extensive progress of isotopic analyzers in near future, we hereafter put to be $1 \times 10^{-9}$ of ${ }^{16} \mathrm{O}$ as the detection limit of rare isotopes.

In the next section, we will briefly present our nuclear reaction network and add a short comment on the reaction rates. In Section 3, we will construct a dynamical model of supernova explosion based on a strong shock propagation in a medium with a power-law density profile as well as a model of neutrino emission and the initial chemical compositions. In Section 4, we will describe the synthetic processes during the supernova explosion for a model of the neutrino emission, which we will call the standard model. In Section 5, we will discuss in detail the influence of the adopted neutrino emission model on the production of light elements, i.e., the influence of the decay time of the neutrino flux and the total energy carried by neutrinos. In Section 6, we will summarize our present numerical simulations and propose three diagrams between two isotopic (elemental) ratios as a 


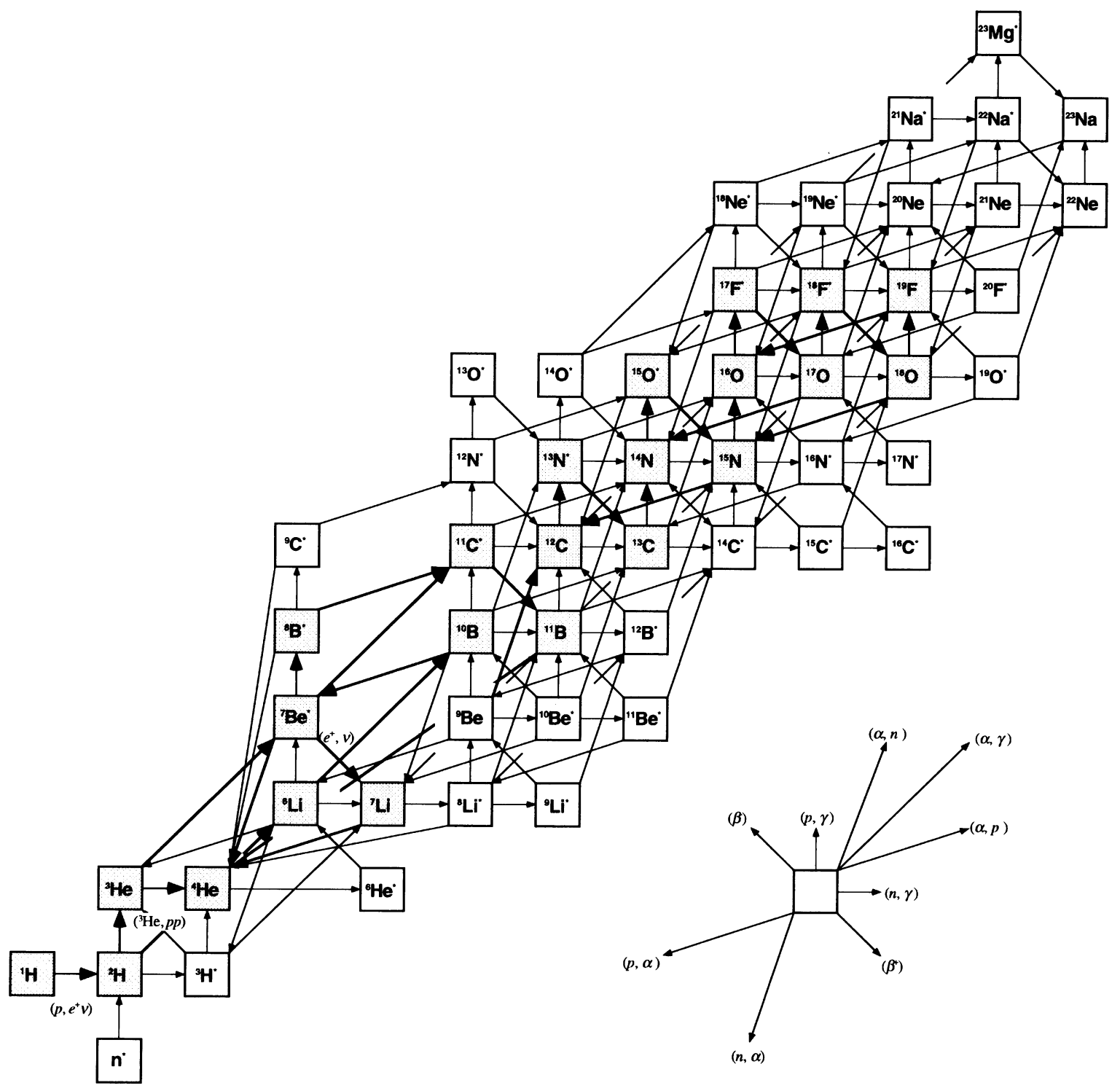

Fig. 1. Isotopic species taken into account in our present study and the nuclear reaction network. Arrows denote the nuclear reactions considered in the present study. A small network, which is composed of shaded isotopic species connected by bold arrows, is used for evaluating the initial chemical composition of light elements in the H-rich envelope. Species with an asterisk are radioactive isotopes.

measure for selection of dust grains of supernova origin (although they are obtained within a framework of our present study).

\section{Nuclear Reaction Network}

In this study, we are interested in the abundances and isotopic ratios of nuclear species from $\mathrm{Li}$ to $\mathrm{O}$ synthesized in the He-layer and the H-rich envelope of a supernova. During the supernova explosion, the maximum temperature of these layers is kept to be lower than the temperature at which the explosive C-burning ignites so that the abundances of elements heavier than Na do not change. Thus, we construct a reaction network including 52 species of elements from $\mathrm{H}$ to $\mathrm{Mg}$, which are illustrated in Fig. 1. We consider various kinds of nuclear reactions in our network: $(n, \gamma)-,(p, \gamma)-,(\alpha, \gamma)$-, $(n, p)-,(n, \alpha)-$, and $(p, \alpha)$-reactions as well as their inverse reactions, $\beta$-decays, and electron captures: we include all reactions relevant not only to the pp chains and the $\mathrm{CNO}$ cy- cles, but also to the hot pp chains, rap-processes (Wiescher et al., 1989), and NeNa cycle (Rolfs and Rodney, 1988). All reactions considered in our present study are tabulated in detail in Yoshida (2000).

Most of the reaction rates are taken from Caughlan and Fowler (1988) which is abbreviated as CF88 hereafter. Some of the rates tabulated in CF88 are reevaluated recently; for such reactions we adopt new ones in our reaction network. For example, for ${ }^{12} \mathrm{C}(\alpha, \gamma){ }^{16} \mathrm{O}$ we use a new rate reevaluated by Buchmann $(1996,1997)$ and for ${ }^{17} \mathrm{O}(p, \alpha){ }^{14} \mathrm{~N}$ and ${ }^{17} \mathrm{O}(p, \gamma){ }^{18} \mathrm{~F}$ we evaluate the reaction rates using the rate formula given by Landré et al. (1990) and the newly found proton width (Blackmon et al., 1995) of $5.673 \mathrm{MeV}$ excited state of ${ }^{18} \mathrm{~F}$. The reaction rate of ${ }^{18} \mathrm{O}(\alpha, \gamma)^{22} \mathrm{Ne}$ is adopted from Käppeler et al. (1994) which includes a resonance of $10.05 \mathrm{MeV}$ of excited state of ${ }^{22} \mathrm{Ne}$. Furthermore, for some reactions which are not listed in CF88, we use the rates presented in other sources (see Yoshida, 2000). 
Table 1. The $\nu$-process reactions. In the table, superscripts * and $\dagger$ represent the $\nu$-processes accompanied with subsequent $\beta^{-}$- and $\beta^{+}$-decays, respectively; e.g., ${ }^{14} \mathrm{O}\left(v, v^{\prime} n\right){ }^{13} \mathrm{O}\left(, e^{+} v_{e}\right){ }^{13} \mathrm{~N}$. Reaction type $\left(v, v^{\prime} \cdot\right)$ includes six kinds of reactions, $\left(v_{e}, v_{e}^{\prime} \cdot\right),\left(\bar{v}_{e}, \bar{v}_{e}^{\prime} \cdot\right),\left(v_{\mu}, v_{\mu}^{\prime} \cdot \cdot\right),\left(\bar{v}_{\mu}, \bar{v}_{\mu}^{\prime} \cdot \cdot\right),\left(v_{\tau}, v_{\tau}^{\prime} \cdot \cdot\right)$, and $\left(\bar{v}_{\tau}, \bar{v}_{\tau}^{\prime} \cdots\right)$. Furthermore, reaction type “others" means $\left(v, v^{\prime 3} \mathrm{He}\right),\left(v, v^{\prime} n \alpha\right),\left(v, v^{\prime} p \alpha\right),\left(v, v^{\prime} p^{3} \mathrm{H}\right),\left(\nu, v^{\prime} n^{3} \mathrm{He}\right)$, and $\left(v, v^{\prime} \alpha \alpha\right)$.

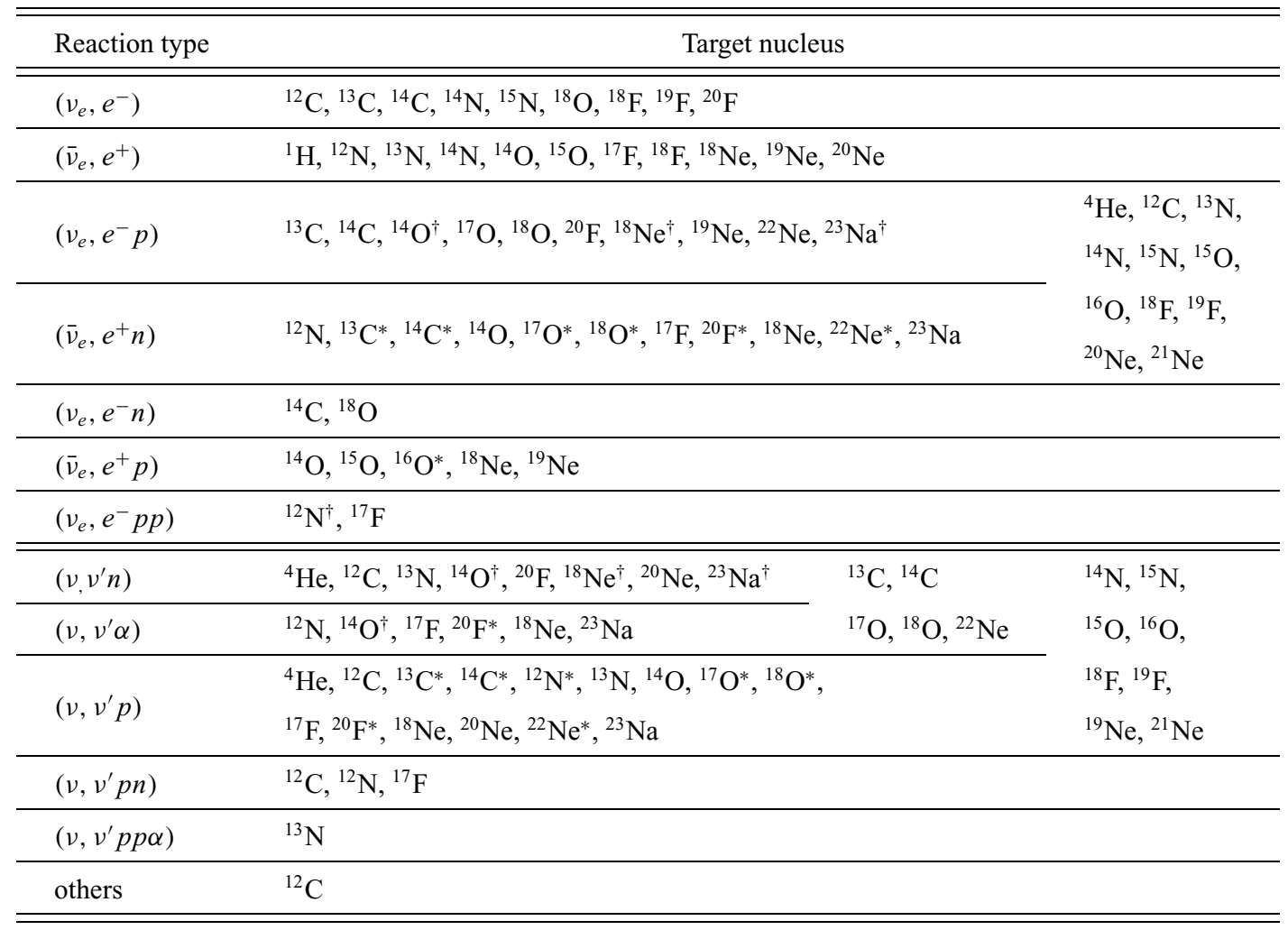

In addition to the ordinary nuclear reactions, the reaction processes induced by high energy neutrinos, which are emitted from the stellar core, play an important role on the light element synthesis (Woosley et al., 1990). Such processes, called the $v$-processes, are also taken into account in our present study. All neutrino reactions in our network are presented in Table 1. For the cross sections and the branching ratios of the $v$-processes we use those given by Hoffman and Woosley (1992). It is worth to note that neutral current reactions dominate the $\nu$-processes and that $\tau$ - and $\mu$-neutrinos with higher energies contribute more than $e$-neutrinos. In order to evaluate the reaction rates of the $v$-processes, it is also necessary to have a knowledge about the mean neutrino temperatures, the number flux of neutrinos, and its time variation. These will be described in Subsection 3.3.

\section{Model of Supernova Explosion}

In a type II supernova, a shock wave generated at the surface of the central core propagates outward and heats up the outer materials. So, nuclear reactions begin newly to work to form various kinds of light elements. Neutrinos and antineutrinos, which are produced in the collapsing central core, induce some nucleosyntheses before the shock arrival and have a good contribution to the synthesis of light elements. In order to pursue such nucleosyntheses, we have to know the neutrino flux, its time variation, the arrival time of the shock wave, and the post shock thermal evolution.

\subsection{Internal stellar model in the presupernova stage}

We construct a stellar model of the presupernova stage (i.e., a model of progenitor) on the basis of model 14E1 obtained numerically by Shigeyama and Nomoto (1990), with the total mass of $16.2 M_{\odot}$. This model corresponds to a $20 M_{\odot}$ main sequence star, which was reduced by $3.8 M_{\odot}$ of mass loss in order to reproduce the light curve properties of SN 1987A. In studying supernova explosions, numerical simulations of supernova explosions with the progenitor's mass of $15 M_{\odot} \sim$ $25 M_{\odot}$ often have been conducted (e.g., Woosley and Weaver, 1995; Meyer et al., 1995; Thielemann et al., 1996). Many numerical simulations of a supernova for comparison with observation of SN 1987A also have been conducted (e.g., Shigeyama et al., 1987; Woosley, 1988; Arnett et al., 1989; Shigeyama and Nomoto, 1990; Thielemann et al., 1990) and the stellar structure of the progenitor was precisely presented in some of them (Shigeyama and Nomoto, 1990). Thus, as the first step of our study, we adopt thermodynamical structure of the progenitor 14E1 model.

In Fig. 2 we show a rough sketch of the outer region of the progenitor together with the radii, $r$, and the internal mass coordinates, $M_{r}$, at the boundaries between layers. The density distribution, $\rho_{0}(r)$, of $14 \mathrm{E} 1$ model is also shown in Fig. 3. For the later convenience, we will approximate $\rho_{0}(r)$ by the power-law:

$$
\rho_{0}(r)=\rho_{00}\left(\frac{r}{r_{0}}\right)^{-\omega} .
$$

Constants $r_{0}, \rho_{00}$ and $\omega$ are determined by fitting the density distribution of the numerical model, 14E1. Fitting is made in a range between the bottom of the Oxygen-layer and a middle position in the H-rich envelope, i.e., from $2.9 \times 10^{8}$ $\mathrm{cm}$ to $1.0 \times 10^{12} \mathrm{~cm}$ (in the outer region where the radius 


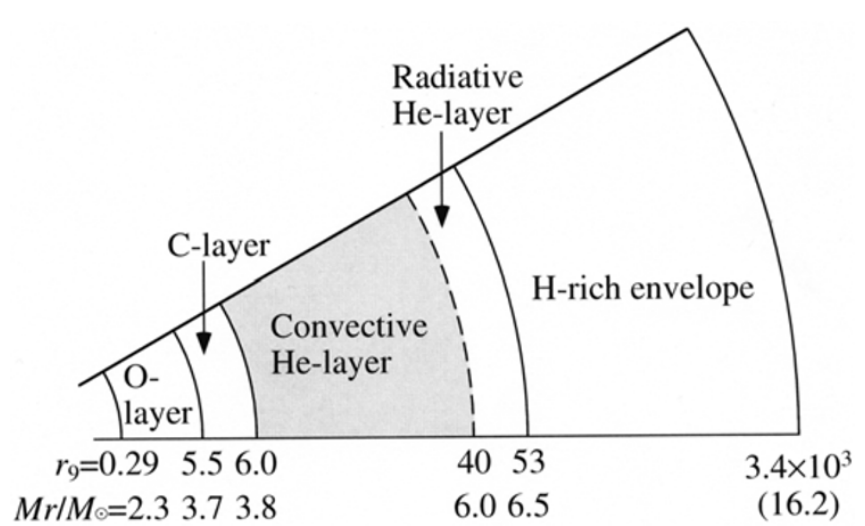

Fig. 2. Schematic picture of the internal stellar structure of the progenitor. Radii, $r_{9}$, and the internal mass coordinates, $M_{r}$, of the boundaries between layers are also shown in the units of $10^{9} \mathrm{~cm}$ and the solar mass, $M_{\odot}$, respectively.

is larger than $1.0 \times 10^{12} \mathrm{~cm}$ the shock wave becomes weak and the temperature does not increase so much; no nuclear processes proceed in this region). As a result we find

$r_{0}=1.0 \times 10^{9} \mathrm{~cm}, \rho_{00}=6.7 \times 10^{4} \mathrm{~g} / \mathrm{cm}^{3}$, and $\omega=2.636$.

The density distribution given by Eqs. (1) and (2) is shown by the dashed line in Fig. 3 together with that of the numerical model 14E1 (the solid line). Note that 14E1 model corresponds to a blue supergiant star. Although a blue supergiant star is small compared with a red supergiant star, the thermodynamical structure is similar between blue and red supergiant stars in the range we fitted.

\subsection{Dynamical model of supernova explosion}

Numerical simulations of the supernova explosions have been carried out by several authors. Some of them mentioned that the radius of a shock front as a function of time can be well approximated by the simple and famous "Sedov solution" in a medium with uniform density distribution (Woosley, 1988; Woosley et al., 1990). Since we are concerned with the outer parts of the stellar interior (i.e., the He-layer and the H-rich envelope), we rather employ another Sedov solution, called the generalized Sedov solution, of a spherically symmetric strong shock wave in a medium with a power law initial density profile (Sedov, 1959). Suppose that an explosion is ignited with energy $E_{0}$ at the center of a spherically symmetric star which is composed of an ideal gas with the ratio of specific heats $\gamma$ and the density distribution of which is given by Eq. (1). According to Sedov, the position of the shock front, $r_{s}$, is described, as a function of time $t$, by

$$
r_{s}=\left(\frac{E_{0} t^{2}}{\rho_{00} r_{0}^{\omega} \alpha}\right)^{1 /(5-\omega)},
$$

where $\alpha$ is a non-dimensional constant determined later from the conservation law of the total energy. By the use of the internal mass $M_{s}$ contained within a sphere of radius $r_{s}$, Eq. (3) can be written as

$$
r_{s}=\frac{1}{\sqrt{(3-\omega) \alpha}}\left(\frac{4 \pi E_{0} t^{2}}{M_{s}}\right)^{1 / 2} .
$$

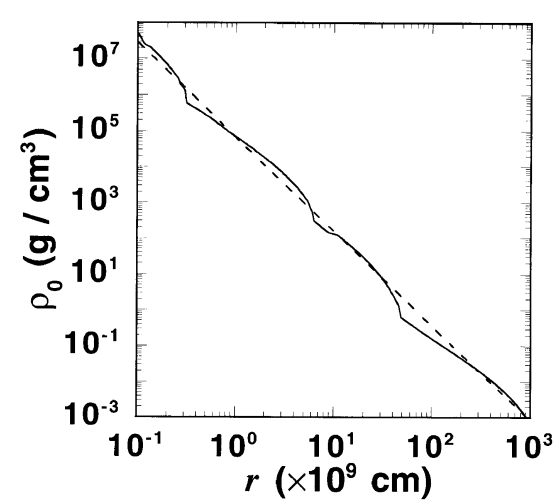

Fig. 3. Stellar density distribution $\rho_{0}(r)$, of the progenitor (after Shigeyama and Nomoto, 1990). The approximate power-law density distribution given by Eqs. (1) and (2) is also shown by the dashed line.

To describe the thermal structure behind the shock front we introduce a non-dimensional variable $\lambda$ instead of $r$ as

$$
\lambda=\frac{r}{r_{s}} \text {. }
$$

For the convenience, we also introduce a variable, $V$, which relates to $\lambda$ as

$$
\begin{aligned}
\lambda^{-1}= & {\left[\frac{(5-\omega)(\gamma+1)}{4} V\right]^{2 /(5-\omega)} } \\
& \times\left[\frac{(5-\omega)(\gamma+1)}{7-\gamma-(\gamma+1) \omega}\left(1-\frac{3 \gamma-1}{2} V\right)\right]^{\alpha_{1}} \\
& \times\left[\frac{\gamma+1}{\gamma-1}\left(\frac{\gamma(5-\omega)}{2} V-1\right)\right]^{\alpha_{2}}
\end{aligned}
$$

where $\alpha_{1}$ and $\alpha_{2}$ are functions of $\omega$ and $\gamma$ (expressions of $\alpha_{1}$ and $\alpha_{2}$ will be given later together with other coefficients). Physically $V$ means the ratio of the characteristic time of expansion at $r_{s}$ and $r$. By the use of dimensionless variables $\lambda$ and $V$, the density behind the shock front is expressed as

$$
\begin{aligned}
\rho= & \frac{\gamma+1}{\gamma-1} \rho_{0} \lambda^{-\omega} \\
& \times\left[\frac{\gamma+1}{\gamma-1}\left(\frac{\gamma(5-\omega)}{2} V-1\right)\right]^{\alpha_{3}} \\
& \times\left[\frac{(5-\omega)(\gamma+1)}{7-\gamma-(\gamma+1) \omega}\left(1-\frac{3 \gamma-1}{2} V\right)\right]^{\alpha_{4}} \\
& \times\left[\frac{\gamma+1}{\gamma-1}\left(1-\frac{5-\omega}{2} V\right)\right]^{\alpha_{5}},
\end{aligned}
$$

and the pressure is given by

$$
\begin{aligned}
P= & \frac{2}{\gamma+1} \rho_{0}\left(\frac{d r_{s}}{d t}\right)^{2}\left[\frac{(5-\omega)(\gamma+1)}{4} V\right]^{6 /(5-\omega)} \\
& \times\left[\frac{(5-\omega)(\gamma+1)}{7-\gamma-(\gamma+1) \omega}\left(1-\frac{3 \gamma-1}{2} V\right)\right]^{\alpha_{4}+\alpha_{1}(\omega-2)} \\
& \times\left[\frac{\gamma+1}{\gamma-1}\left(1-\frac{5-\omega}{2} V\right)\right]^{\alpha_{5}+1}
\end{aligned}
$$

where $d r_{s} / d t$ is the propagating speed of the shock front. Coefficients $\alpha_{1}$ to $\alpha_{5}$ used in the above expressions are defined 
as follows (Sedov, 1959):

$$
\begin{aligned}
\alpha_{1} & =\frac{(5-\omega) \gamma}{3 \gamma-1}\left[\frac{2(6-3 \gamma-\omega)}{\gamma(5-\omega)^{2}}-\frac{1-\gamma}{2 \gamma+1-\gamma \omega}\right] \\
\alpha_{2} & =\frac{1-\gamma}{2 \gamma+1-\gamma \omega} \\
\alpha_{3} & =\frac{-\omega+3}{2 \gamma+1-\gamma \omega} \\
\alpha_{4} & =\frac{(5-\omega)(3-\omega) \alpha_{1}}{6-3 \gamma-\omega}
\end{aligned}
$$

and

$$
\alpha_{5}=\frac{\omega(1+\gamma)-6}{6-3 \gamma-\omega}
$$

In an exploding supernova, the pressure behind the shock front is almost determined by the radiation pressure owing to the high temperature (Weaver and Woosley, 1980). So the ratio of specific heats $\gamma$ can be put to be $4 / 3$, that of the fluid perfectly supported by photon pressure and, thus, the temperature behind the shock front is written by

$$
T=\left(\frac{3 P}{a}\right)^{1 / 4}
$$

where $a$ is the radiation density constant.

The constant $\alpha$ introduced in Eq. (3) is determined by the condition that the sum of thermal energy and kinetic energy inside the shock front is equal to the initial explosion energy $E_{0}$ :

$$
\begin{aligned}
\alpha= & \int_{0}^{1}\left[\frac{1}{2} \frac{\rho}{\rho_{0}} \lambda^{2} V^{2}+\frac{2}{(\gamma+1)(\gamma-1)}\left(\frac{2}{5-\omega}\right)^{2}\right. \\
& \left.\times P\left\{\frac{2}{\gamma+1} \rho_{0}\left(\frac{d r_{s}}{d t}\right)^{2}\right\}^{-1}\right] 4 \pi \lambda^{2} d \lambda .
\end{aligned}
$$

According to Shigeyama and Nomoto (1990), the explosion energy $E_{0}$ is put to be $1 \times 10^{51} \mathrm{erg}$. Using the above equations we obtain

$$
\alpha=9.96 \text {. }
$$

In order to confirm the validity of our explosion model, we checked the propagation of the shock profile, which is evaluated from our simple model, with that of the 14E1 model. As a whole, the evaluated profiles coincide well with those of the 14E1 model. However, at the regions just outside the boundaries between the C-layer and the He-layer and between the He-layer and the H-rich envelope, the differences become relatively large; especially the evaluated shock density is larger by a factor of 3 or 4 compared to that of 14E1 model. Nevertheless, we do not pay special attention to the density difference mentioned above because we know that the material density does not play any important roles on the nucleosynthesis. The evaluated temperature is higher by $30 \%$ or so at the regions just outside the boundaries. In order to see how the inaccurate model temperature affects the final result of isotopic ratios in which we are interested (see Section 6), we calculated, as a test case, the explosive nucleosynthesis at the bottom of the He-layer $\left(r=6.0 \times 10^{9} \mathrm{~cm}\right)$ assuming that the temperature during the explosion is decreased by $30 \%$ artificially. By the test calculation we found that the isotopic ratios can be reproduced properly within a factor of 3. The difference is not so important because in our study we take notice on the isotopic ratios of light elements in order of magnitude.

\subsection{Model of neutrino emission}

In the present study, we adopt a model of the flux evolution of neutrinos and antineutrinos proposed by Woosley et al. (1990), in which there are eight parameters, i.e., the total energy carried by neutrinos, $E_{v}$, the temperature of each neutrino flavor, $T_{v i}$, and the decay time of the neutrino flux, $\tau_{v}$.

A core collapse in a type II supernova liberates an enormous amount of gravitational energy, most of which is carried by neutrinos and antineutrinos. So the total energy carried by neutrinos is almost equal to the gravitational binding energy of a resulting neutron star. From these reasons, Woosley et al. evaluated $E_{v}$ to be $3 \times 10^{53}$ erg based on a neutron star model proposed by Cooperstein (1988). Cooperstein also showed that one half of the total neutrino energy is released within the first $2 \mathrm{~s}$ and most of the rest energy comes out over the next $10 \mathrm{~s}$. On the basis of his results, Woosley et $a l$. adopted a model that the number flux of $i$-flavor neutrino, $F_{i}$, decreases exponentially with a decay time, $\tau_{v}=3 \mathrm{~s}$ :

$$
F_{i}=\frac{1}{6} \frac{1}{4 \pi r^{2}} \frac{E_{v}}{\varepsilon_{i}} \frac{1}{\tau_{v}} e^{-\frac{t-r / c}{\tau_{v}}} \Theta(t-r / c),
$$

where $\Theta(x)$ is the step function $(\Theta(x)=1$ for $x>0$ and 0 otherwise) and $\varepsilon_{i}$ is the mean energy per particle of $i$-flavor neutrino, $\varepsilon_{i}=3.15 T_{v i}$ (Woosley et al., 1990). According to Woosley et al. (1990), $E_{v}$ and $\tau_{v}$ are put to be $3 \times 10^{53}$ erg and $3 \mathrm{~s}$, respectively, as standard values. It should be noted that there are yet uncertainties in the equation of state of neutron star matter as well as the mass of the neutron star (e.g., Lattimer and Yahil, 1989) and that $\tau_{v}$ is determined by the diffusion transport which is affected in a complicated manner by the physical conditions of the deep stellar interior. So it is not always certain whether the above values of $E_{v}$ and $\tau_{v}$ are proper or not. Taking account of the possible mass range of a neutron star between 1 and $2 M_{\odot}$ (Bahcall, 1989), we consider the following three cases for $E_{\nu}$ :

$$
E_{v}=1 \times 10^{53} \mathrm{erg}, \quad 3 \times 10^{53} \mathrm{erg}, \quad \text { and } 6 \times 10^{53} \mathrm{erg} .
$$

According to the textbook of Bahcall (1989), the characteristic time of the neutrino irradiation is in a range between $1 \mathrm{~s}$ and $10 \mathrm{~s}$. In fact, Spergel et al. (1987) evaluated the decay time of the neutrino flux as $4.5 \pm 2 \mathrm{~s}$ on the basis of the observation of SN1987A. Thus, for $\tau_{v}$ we consider the three cases where

$$
\tau_{v}=1 \mathrm{~s}, \quad 3 \mathrm{~s}, \quad \text { and } 10 \mathrm{~s} .
$$

Woosley et al. (1990) estimated theoretically neutrino temperatures and obtained the result with some uncertainties that temperatures of $e^{-}, \mu-$, and $\tau$-neutrinos are $T_{v e} \simeq 4$ to 5 $\mathrm{MeV} / k$ ( $k$ being the Boltzmann constant), $T_{\nu \mu} \simeq 8$ to 10 $\mathrm{MeV} / k$, and $T_{\nu \tau} \simeq 8$ to $10 \mathrm{MeV} / k$, respectively. Considering the observational data of $1987 \mathrm{~A}$ which suggest $T_{v e} \simeq 3$ to 4 
Table 2. Initial mass fractions of the most abundant isotopes.

\begin{tabular}{cccc}
\hline Species & Convective He-layer & Radiative He-layer & H-rich envelope \\
\hline${ }^{1} \mathrm{H}$ & - & $1.0 \times 10^{-5}$ & 0.565 \\
${ }^{4} \mathrm{He}$ & 0.930 & 0.995 & 0.430 \\
${ }^{12} \mathrm{C}$ & $6.2 \times 10^{-2}$ & $8.5 \times 10^{-5}$ & $8.4 \times 10^{-5}$ \\
${ }^{14} \mathrm{~N}$ & $5.8 \times 10^{-8}$ & $4.8 \times 10^{-3}$ & $4.8 \times 10^{-3}$ \\
${ }^{16} \mathrm{O}$ & $7.2 \times 10^{-4}$ & $1.0 \times 10^{-4}$ & $1.0 \times 10^{-4}$ \\
\hline
\end{tabular}

$\mathrm{MeV} / k$ (Burrows and Lattimer, 1987), they used the smallest values estimated theoretically. Although there are still uncertainties on the neutrino temperatures, we also adopt the same values as Woosley et al. (1990) in the present study:

$$
\begin{aligned}
& T_{\nu e}=T_{\bar{\nu} e}=4 \mathrm{MeV} / k \quad \text { and } \\
& T_{\nu \mu}=T_{\bar{\nu} \mu}=T_{\nu \tau}=T_{\bar{\nu} \tau}=8 \mathrm{MeV} / k .
\end{aligned}
$$

We do not adopt the influence of neutrino temperatures. Woosley et al. (1990) investigated sensitivity of the $X$-element production to $T_{v i}$. Their result shows that the variety of the amounts of the $X$-elements is within a factor of 3 . As we will present in Section 5, the variety due to the uncertainties of $\tau_{v}$ and $E_{v}$ is within five orders of magnitude, so that the variety due to $T_{\nu i}$ is expected to be much smaller. On the other hand, Myra and Burrows (1990) indicated that the peak and the high-energy tails of neutrino spectra of all species are fitted by thermal distributions having nonzero chemical potentials, but again, Woosley et al. (1990) varied the temperature to test the sensitivity to the chemical potentials; the varieties are small, as mentioned above.

\subsection{Initial chemical compositions}

The chemical compositions just before the supernova explosion are generally to be determined as a result of the evolution of its progenitor. Until now, many works were made on the evolution of progenitors of supernovae (e.g., Woosley and Weaver, 1986; Nomoto and Hashimoto, 1988). In such works, however, some authors concentrated on special supernova events and other authors did not take account of nuclear species which play an important role on our study. So, in the present study, we prepare by ourselves the chemical compositions just before the supernova explosion by the following way.

It should be noted that the chemical compositions in the H-rich envelope depend strongly on whether the convective region develops in the envelope or not. Lamb et al. (1976) pursued the evolution of a $25 M_{\odot}$ star after the main sequence stage and showed that there is a convective region in the inner part of the H-rich envelope. Furthermore, Saio et al. (1988) pursued the evolution of the progenitor of SN1987A and showed that a large scale convection occurs in the H-rich envelope. So we expect that there is a convective region in the inner H-rich envelope of a 16.2 $M_{\odot}$ star. In the convective region, the chemical compositions would be determined by the steady-flow equilibrium condition of the H-burning. The equilibrium chemical abundance is calculated by using a small reaction network composed of shaded nuclear species in Fig. 1. The temperature and the density at the bottom of the
H-rich envelope are, respectively, taken to be $4 \times 10^{7} \mathrm{~K}$ and $5 \mathrm{~g} / \mathrm{cm}^{3}$ after Lamb et al. (1976) and the mass fractions of ${ }^{1} \mathrm{H}$ and ${ }^{4} \mathrm{He}$ put to be 0.565 and 0.430 , respectively (Shigeyama and Nomoto, 1990). For isotopes which do not take part in the small reaction network (i.e., unshaded isotopes in Fig. 1), the mass fractions are set artificially to be $1 \times 10^{-50}$, which is the smallest value in our calculation. Note that we adopt ${ }^{7} \mathrm{Be}$ abundance in the steady-flow equilibrium as initial ${ }^{7} \mathrm{Li}$ abundance in the $\mathrm{H}$-rich envelope and initial ${ }^{7} \mathrm{Be}$ abundance is set to be $1 \times 10^{-50}$ because it is expected that ${ }^{7} \mathrm{Be}$ produced in the H-burning shell is electron-captured to ${ }^{7} \mathrm{Li}$ in outer and cooler part of the H-rich envelope. Although the abundances of ${ }^{1} \mathrm{H}$ and ${ }^{4} \mathrm{He}$, exactly speaking, change in the course of the $\mathrm{H}$-shell burning, the steady-flow equilibrium abundances of the CNO-elements are hardly affected by the ${ }^{1} \mathrm{H}$ and ${ }^{4} \mathrm{He}$ abundances. The abundances of the $X$-elements (i.e., Li, Be, and B) vary with the ${ }^{4} \mathrm{He}$ abundance almost proportionally. However, these variations are not important because their abundances are very small compared with those produced by the explosive nucleosynthesis.

Shigeyama and Nomoto (1990) studied the chemical compositions in the He-layer of a He star (corresponding to a 16.2 $M_{\odot}$ star) modeled by Nomoto and Hashimoto (1988). Unfortunately, they did not give a detailed description on the time variation of the chemical composition nor take account of some species in which we are interested. So we prepare the chemical compositions in the progenitor according to the model of a He star of Nomoto and Hashimoto. In this model the inner region of the He-layer $\left(3.8 M_{\odot} \lesssim M_{r} \lesssim 6.0 M_{\odot}\right)$ is convective and the outer region $\left(6.0 M_{\odot} \lesssim M_{r} \lesssim 6.5 M_{\odot}\right)$ is radiative. In the outer radiative region, the chemical compositions would be determined originally at the stage of the H-burning and may not suffer from further nuclear burning processes. So, as the initial chemical compositions in this region, we adopt those of ashes of the H-burning; the chemical compositions are evaluated by solving the nuclear reaction network until the mass fraction of ${ }^{1} \mathrm{H}$ decreases to $1 \times 10^{-5}$ (e.g., Woosley et al., 1990) under the same physical condition at the bottom of the H-rich envelope. For the compositions in the inner convective region of the He-layer, we adopt those of the He-burning products when the mass fraction of ${ }^{4} \mathrm{He}$ decreases to 0.930 (i.e., when the stellar core begins to collapse (Nomoto and Hashimoto, 1988)). The chemical compositions are calculated by using our nuclear reaction network (see Fig. 1). The obtained initial mass fractions of the most abundant (key) species are tabulated in Table 2 and those of important species are illustrated in Fig. 4. Note that the 

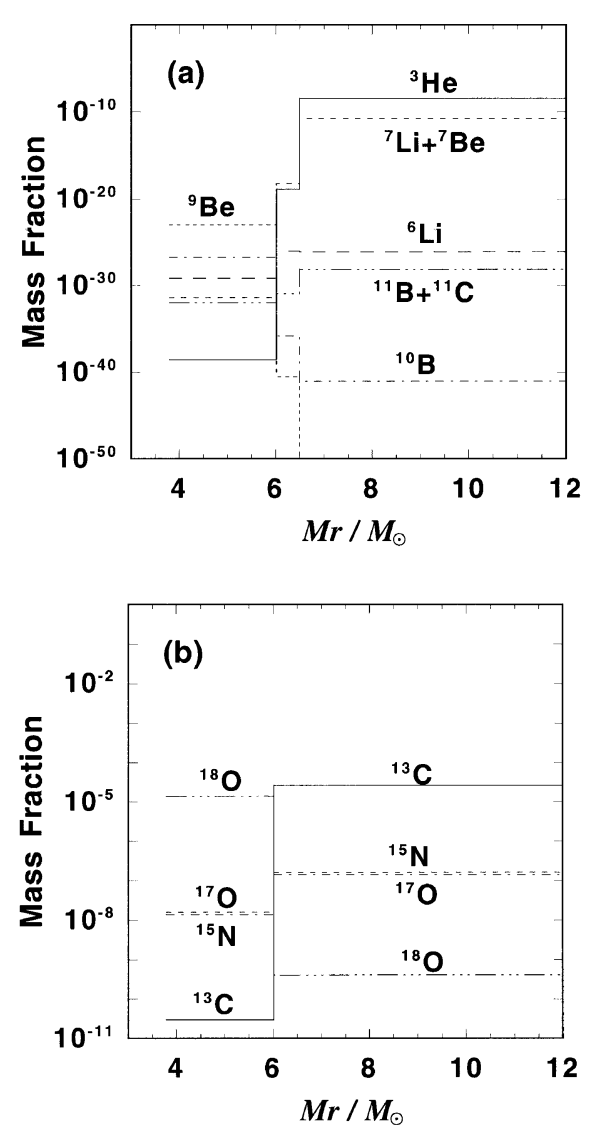

Fig. 4. Initial mass fractions of important isotopes. Horizontal axis shows the internal mass coordinates, $M_{r}$, in the unit of the solar mass, $M_{\odot}$. For the most abundant isotopes, ${ }^{1} \mathrm{H},{ }^{4} \mathrm{He},{ }^{12} \mathrm{C},{ }^{14} \mathrm{~N}$, and ${ }^{16} \mathrm{O}$, the initial mass fractions are tabulated in Table 2 separately.

abundances of $X$-elements are very little in the whole regions of the He-layer and the H-rich envelope.

The initial chemical compositions are different from those of Woosley et al. (1990). After Woosley et al. (1990), some reaction rates, especially concerned to oxygen, are updated as mentioned in Section 2. As a result, the initial amounts of the CNO-elements should be recalculated. Convective mixing during the stellar evolution is also expected to affect the initial composition, so that we include this effect for determining it.

\section{Light Element Synthesis in the Case of the Stan- dard Model}

The amounts of isotopes synthesized in the supernova explosion depend, by a complicated manner, on the physical parameters characterizing the neutrino emission and the dynamics of the supernova explosion as well as the temporal and spatial points we observe. In this section, to grasp its general features, we will show briefly the isotopic synthesis in the He-layer and the H-rich envelope of the supernova only in the case of the standard model where we put $E_{v}=3 \times 10^{53}$ erg and $\tau_{v}=3 \mathrm{~s}$ for the neutrino emission model.

\subsection{General behavior of the $X$-element synthesis}

In Fig. 5, we illustrate the time variation of the amount of ${ }^{7} \mathrm{Li}$ for typical internal mass coordinates. Although, at a glance, the abundance curves behave quite differently from

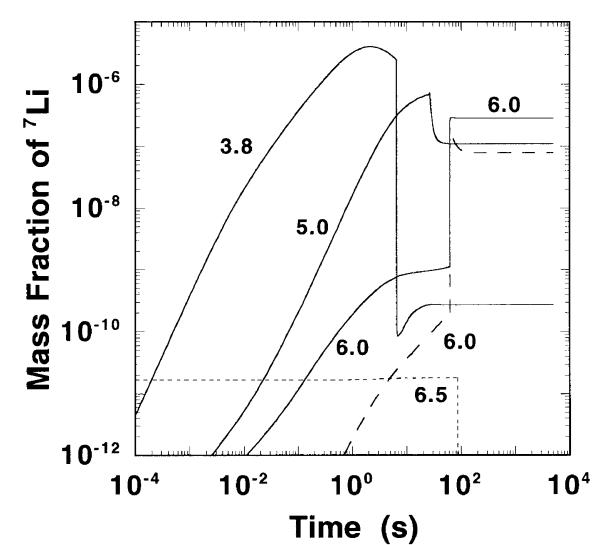

Fig. 5. Time variation of the amount of ${ }^{7} \mathrm{Li}$. Solid lines are of the time evolution in the convective He-layer. Attached numbers indicate the internal mass coordinates of each point. Dashed and dotted lines show the time evolutions at the bottom of the radiative He-layer and at the bottom of the H-rich envelope, respectively.

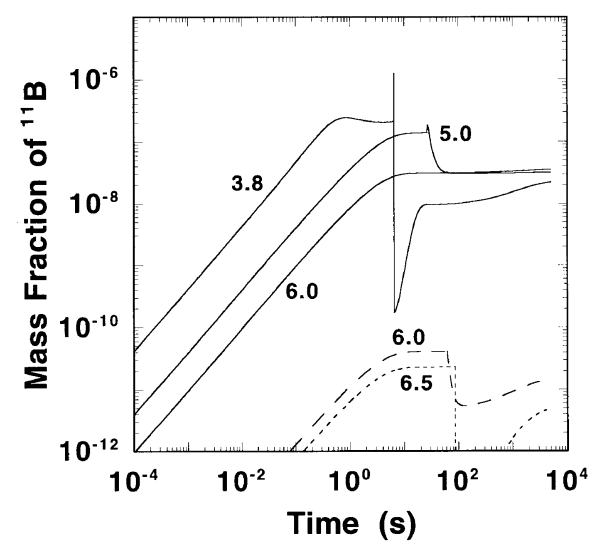

Fig. 6. The same as Fig. 5, but for ${ }^{11} \mathrm{~B}$.

point to point of the internal mass coordinates, the abundance evolution is generally divided into three stages: the preshock stage, the shock-induced (explosive) burning stage, and the freezing stage. In the first stage (i.e., the preshock stage) before the arrival of the explosive shock wave, $X$-elements are synthesized through various kinds of the $v$-processes and subsequent nuclear reactions. For ${ }^{7} \mathrm{Li},{ }^{4} \mathrm{He}\left(v, v^{\prime} p\right){ }^{3} \mathrm{H}(\alpha, \gamma){ }^{7} \mathrm{Li}$ and $/$ or $^{4} \mathrm{He}\left(\nu, v^{\prime} n\right)^{3} \mathrm{He}(\alpha, \gamma)^{7} \mathrm{Be}\left(e^{-}, v_{e}\right)^{7} \mathrm{Li}$ are the major formation reactions in the He-layer. In the inner region $\left(M_{r} \lesssim\right.$ $\left.5 M_{\odot}\right)$, the neutrino flux is so intensive that the large amount of ${ }^{7} \mathrm{Li}$ is synthesized. In the outer region the amount of synthesized ${ }^{7} \mathrm{Li}$ decreases with a decrease in the neutrino flux and ${ }^{7} \mathrm{Li}$ is hardly formed near the bottom of the H-rich envelope (i.e., at $M_{r}=6.5 M_{\odot}$ ), since neutrino flux is $1.3 \times 10^{-2}$ times as small as that at the innermost region of the He-layer (i.e., at $M_{r}=3.8 M_{\odot}$ ).

The second (the shock-induced burning) stage starts at the moment of the shock arrival and ends when the temperature becomes sufficiently low owing to the expansion accompanied with the adiabatic cooling. By a sudden increase in temperature, new channels of nuclear reactions open; some isotopes which are synthesized by the $v$-processes are decomposed and some isotopes are newly formed through various 


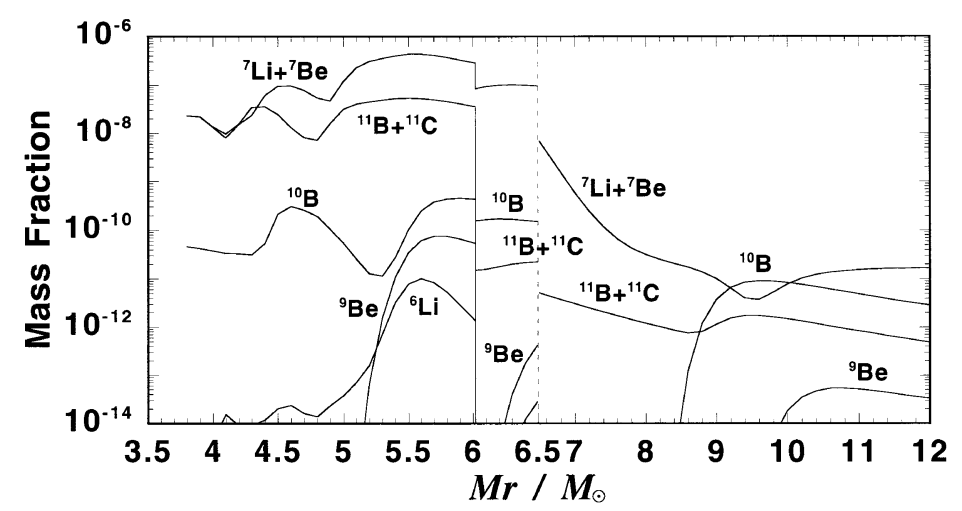

Fig. 7. Distribution of the final abundances of the $X$-elements. Discontinuities of the curves appear at the boundaries between the convective and the radiative He-layers $\left(M_{r}=6.0 M_{\odot}\right)$ and between the radiative He-layer and the H-rich envelope $\left(M_{r}=6.5 M_{\odot}\right)$.

Table 3. Typical key reactions of light element synthesis. The upper and lower columns show the formation and decomposition reactions, respectively. Furthermore, empty columns mean that there are no appreciable formation (or decomposition) processes.

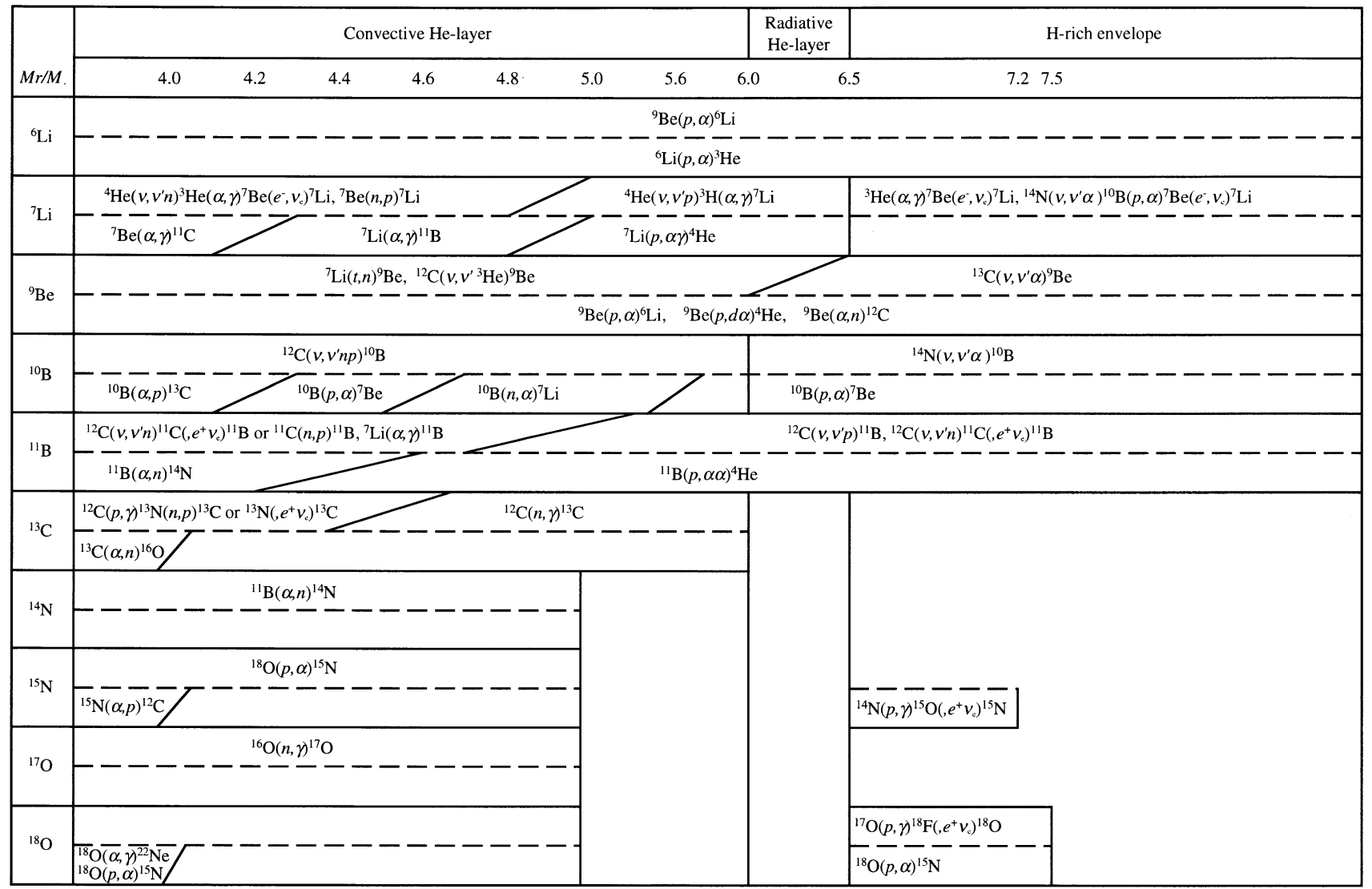

kinds of nuclear reactions. For example, ${ }^{7} \mathrm{Li}$ produced by the $\nu$-process is destroyed abruptly and almost completely through ${ }^{7} \mathrm{Li}(\alpha, \gamma){ }^{11} \mathrm{~B}$ in the innermost region whereas ${ }^{7} \mathrm{Li}$ is formed by ${ }^{3} \mathrm{H}(\alpha, \gamma){ }^{7} \mathrm{Li}$ in the outer region. In the last stage where the temperature is too low, there occurs no nuclear reactions except the beta processes and the abundance is frozen to the level of the end of the second stage if no beta processes play an important role.

Similar behavior is seen from Fig. 6 where the abundance evolution of ${ }^{11} \mathrm{~B}$ is shown. Spikes observed at $t=7 \mathrm{~s}$ $\left(M_{r}=3.8 M_{\odot}\right)$ and $t=27 \mathrm{~s}\left(M_{r}=5.0 M_{\odot}\right)$ are the result of the sudden production of ${ }^{11} \mathrm{~B}$ through ${ }^{7} \mathrm{Li}(\alpha, \gamma){ }^{11} \mathrm{~B}$, as mentioned earlier, just after the shock arrival and of the rapid disruption due to ${ }^{11} \mathrm{~B}(p, \alpha \alpha){ }^{4} \mathrm{He}$ and ${ }^{11} \mathrm{~B}(\alpha, n){ }^{14} \mathrm{~N}$. In the case of ${ }^{11} \mathrm{~B}$, the abundance increase gradually even in the third stage. This is due to the $\beta^{+}$-decay of ${ }^{11} \mathrm{C}$ with the decay time of $1.8 \times 10^{3} \mathrm{~s}$.

As seen from Figs. 5 and 6, the amounts of $X$-elements vary from point to point of the internal mass coordinates. The distribution of the final abundances of $X$-elements are illustrated in Fig. 7. The final abundance depends complicatedly on the initial chemical composition, neutrino flux, and time of the shock arrival. In the He-layer, the most abundant $X$-elements are ${ }^{7} \mathrm{Li}$ and ${ }^{11} \mathrm{~B}$. Production of all 
$X$-elements (as well as ${ }^{11} \mathrm{C}$ ) is initiated by the $v$-processes, ${ }^{4} \mathrm{He}+v$ and ${ }^{12} \mathrm{C}+v$, and the amounts produced during the preshock stage are almost same. However, after the shock passage, they are recooked by hot nuclear reactions; especially, ${ }^{6} \mathrm{Li}$ and ${ }^{9} \mathrm{Be}$ are disrupted almost completely. In the $\mathrm{H}$-rich envelope (but $M_{r} \lesssim 8.0 M_{\odot}$ ), only ${ }^{7} \mathrm{Li}$ is synthesized through ${ }^{4} \mathrm{He}\left(\nu, v^{\prime} n\right)^{3} \mathrm{He}(\alpha, \gamma)^{7} \mathrm{Be}\left(e^{-}, v_{\mathrm{e}}\right)^{7} \mathrm{Li}$ by an appreciable amount. The production of the other $X$-elements are inactive in the H-rich envelope: their mass fractions are lower than a level of $1 \times 10^{-10}$.

Key reactions (production and decomposition reactions) which play important roles on determining the abundances of light elements are shown in Table 3. As a matter of course, key reactions vary from point to point as well as from time to time so that in the table only typical reactions are tabulated. In the preshock stage, the $X$-elements are synthesized, mainly, through the $v$-processes with the target nuclei of ${ }^{4} \mathrm{He}$ and ${ }^{12} \mathrm{C}$. In the shock-induced burning stage, various kinds of nuclear reactions take part in the formation and destruction of the $X$-elements.

Comparing with the result of Woosley et al. (1990) or a $25 M_{\odot}$ supernova model in Woosley and Weaver (1995), the difference of the amounts of $\mathrm{Li}$ and $\mathrm{B}$ are within two orders of magnitude. Roughly speaking, our result is more similar to that of Woosley and Weaver (1995). As mentioned in Section 2, some reaction rates are updated from those of Woosley et al. (1990) and Woosley and Weaver (1995). As a result, chemical compositions in the presupernova stage and production and decomposition processes are changed. For example, since in the convective He-layer the amount of ${ }^{18} \mathrm{O}$ is three orders of magnitude less than that in Woosley et al. (1990), ${ }^{18} \mathrm{O}$ does not fill a role as proton-poison. Hence, ${ }^{11} \mathrm{~B}$ is decomposed by protons and the amount decreases compared with those in Woosley et al. (1990) and Woosley and Weaver (1995).

\section{2 ${ }^{6} \mathrm{Li}$ and ${ }^{9} \mathrm{Be}$ production}

As mentioned briefly in the previous subsection, ${ }^{6} \mathrm{Li}$ and ${ }^{9} \mathrm{Be}$ are scarcely produced during the supernova explosion. Here we will see in detail the formation processes of these two isotopes and discuss the reasons of the rare production.

In the He-layer ${ }^{6} \mathrm{Li}$ is commonly produced through ${ }^{9} \mathrm{Be}(p, \alpha)^{6} \mathrm{Li}$. Nevertheless, we cannot find the curve of ${ }^{6} \mathrm{Li}$ in Fig. 7 except the narrow region where $5.3 M_{\odot} \lesssim M_{r} \lesssim$ $6.0 M_{\odot}$. In the inner region where $M_{r} \lesssim 5.3 M_{\odot}{ }^{6} \mathrm{Li}$ is formed by an appreciable amount (say, a level of $3 \times 10^{-10}$ by mass fraction) owing to the neutrino irradiation. However, as soon as the shock front reaches ${ }^{6} \mathrm{Li}$ is completely decomposed through ${ }^{6} \mathrm{Li}(p, \alpha)^{3} \mathrm{He}$. In the outer region where $5.3 M_{\odot} \lesssim M_{r} \lesssim 6.0 M_{\odot}$, a little amount of ${ }^{6} \mathrm{Li}$ can survive because the temperature is not so high (compared with that in the inner region) even just after the passage of the explosive shock. In the radiative He-layer and the H-rich envelope ${ }^{6} \mathrm{Li}$ is produced and decomposed through the same processes as those in the convective He-layer. However, in these regions the initial abundance of ${ }^{12} \mathrm{C}$, which is a seed isotope of ${ }^{6} \mathrm{Li}$, is very low (about three orders of magnitude below) and the neutrino flux dilutes by about two orders of magnitude compared with those in the inner convective region. As a result, the amount of produced ${ }^{6} \mathrm{Li}$ is suppressed under the low level. Furthermore, ${ }^{6} \mathrm{Li}$ (as well as ${ }^{7} \mathrm{Li}$ ) is hardly produced (nor de-
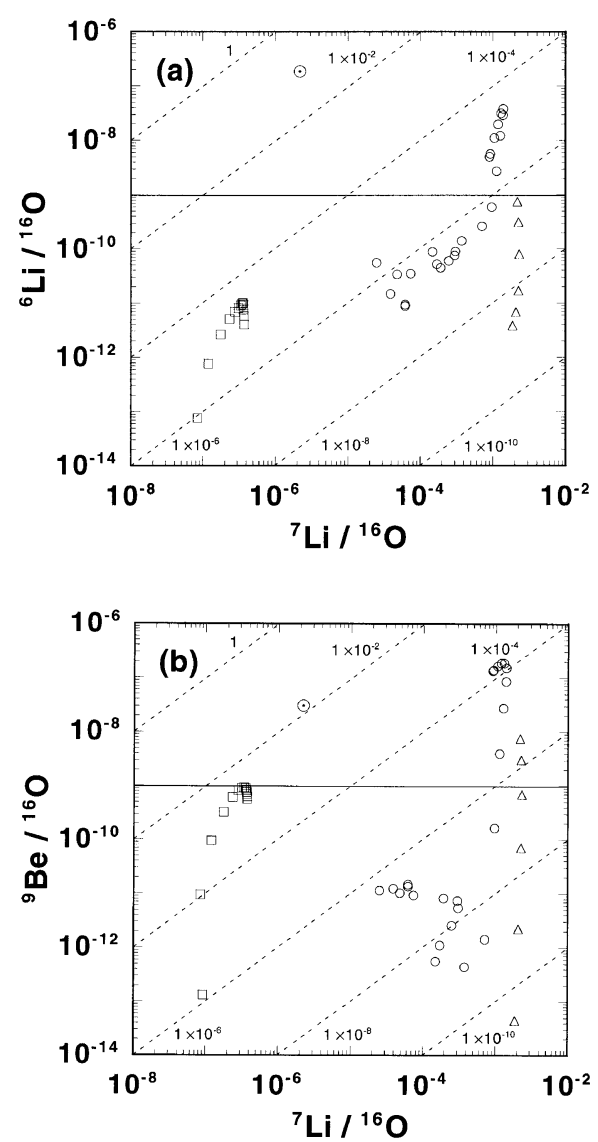

Fig. 8. Diagrams between ${ }^{6} \mathrm{Li} /{ }^{16} \mathrm{O}$ and ${ }^{7} \mathrm{Li} /{ }^{16} \mathrm{O}$ ratios (panel (a)) and between ${ }^{9} \mathrm{Be} /{ }^{16} \mathrm{O}$ and ${ }^{7} \mathrm{Li} /{ }^{16} \mathrm{O}$ ratios (panel $(b)$ ). The ratios are all in number. Circles, triangles, and squares show the ratios in the convective He-layer, in the radiative He-layer, and in the H-rich envelope, respectively. A mark $\odot$ shows the ratios of solar-system composition. A solid horizontal line indicates the detection limit, i.e., the number ratio of species to ${ }^{16} \mathrm{O}$ equal to $1 \times 10^{-9}$. Furthermore, dotted lines indicate values of constant ${ }^{6} \mathrm{Li} /{ }^{7} \mathrm{Li}$ ratio (a) and ${ }^{9} \mathrm{Be} /{ }^{7} \mathrm{Li}$ ratio (b): $1,1 \times 10^{-2}, 1 \times 10^{-4}, 1 \times 10^{-6}$, $1 \times 10^{-8}$ and $1 \times 10^{-10}$.

composed) in the outermost H-rich envelope $\left(M_{r} \gtrsim 11 M_{\odot}\right)$ since the shock temperature is very low and the neutrino flux is very weak. Thus, the amount of produced ${ }^{6} \mathrm{Li}$ is less than $1 \times 10^{-15}$ by mass fraction throughout the H-rich envelope.

For ${ }^{9} \mathrm{Be}$, the situation is quite the same as in the case of ${ }^{6} \mathrm{Li}$. In the convective He-layer, ${ }^{9} \mathrm{Be}$ is produced through ${ }^{12} \mathrm{C}\left(v, v^{\prime 3} \mathrm{He}\right){ }^{9} \mathrm{Be}$ in the preshock stage, ${ }^{7} \mathrm{Li}(t, n){ }^{9} \mathrm{Be}$ in the shock-induced burning stage. In the radiative He-layer and the H-rich envelope, ${ }^{13} \mathrm{C}\left(v, v^{\prime} \alpha\right){ }^{9} \mathrm{Be}$ is a dominant reaction in the preshock stage. In the He-layer and the H-rich envelope, ${ }^{9} \mathrm{Be}$ is commonly decomposed through ${ }^{9} \mathrm{Be}(p, \alpha){ }^{6} \mathrm{Li}$, ${ }^{9} \mathrm{Be}(p, n \alpha){ }^{4} \mathrm{He}$ or ${ }^{9} \mathrm{Be}(\alpha, n){ }^{12} \mathrm{C}$ in the shock-induced burning stage. With an increase in the radius in the convective He-layer, the amount of ${ }^{9} \mathrm{Be}$ firstly becomes larger owing to less decomposition and becomes smaller again since the neutrino flux becomes smaller owing to the geometrical effect. As in the case of ${ }^{6} \mathrm{Li}$, the amount of ${ }^{9} \mathrm{Be}$ becomes appreciable only in the outer region where $5.3 M_{\odot} \lesssim M_{r} \lesssim 6.0 M_{\odot}$ in the He-layer.

In Figs. 8(a) and 8(b), the same results (but only ${ }^{6} \mathrm{Li}$, ${ }^{7} \mathrm{Li}$, and ${ }^{9} \mathrm{Be}$ ) as in Fig. 7 are transferred to the diagrams of ${ }^{6} \mathrm{Li} /{ }^{16} \mathrm{O}-{ }^{7} \mathrm{Li} /{ }^{16} \mathrm{O}$ and ${ }^{9} \mathrm{Be} /{ }^{16} \mathrm{O}-{ }^{7} \mathrm{Li} /{ }^{16} \mathrm{O}$ where ${ }^{i} \mathrm{X} /{ }^{16} \mathrm{O}$ is the 


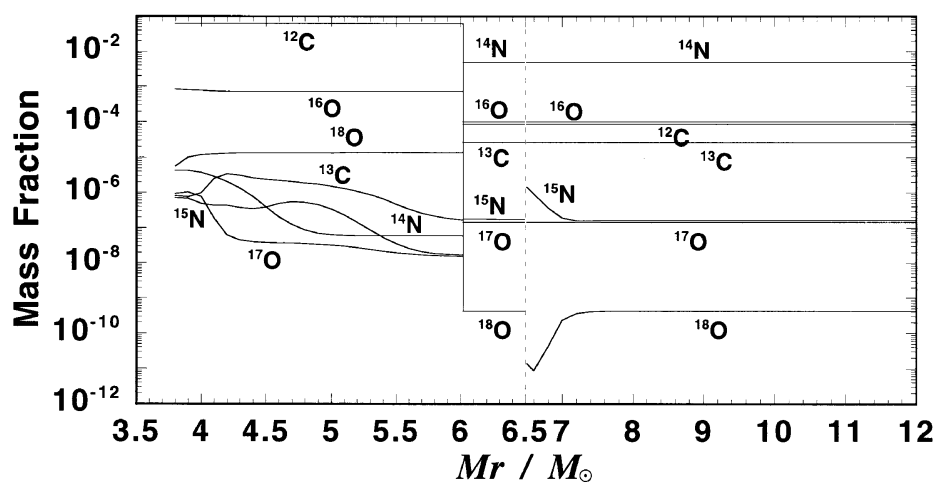

Fig. 9. The same as Fig. 7, but for the CNO-elements.

number ratio of ${ }^{i} \mathrm{X}$-isotope synthesized in the supernova explosion to ${ }^{16} \mathrm{O}$. We can see clearly that ${ }^{6} \mathrm{Li}$ produced in the supernova explosion is very small compared with the number of ${ }^{16} \mathrm{O}$; only in the narrow region where $5.3 M_{\odot} \lesssim M_{r} \lesssim 6.0 M_{\odot}$ (see Fig. 7) we have ${ }^{6} \mathrm{Li} /{ }^{16} \mathrm{O}$ greater than the detection limit, $1 \times 10^{-9}$ (see Introduction), and we can never detect ${ }^{6} \mathrm{Li}$ produced in the other places. It should be also noted that, even if we can observe a trace of ${ }^{6} \mathrm{Li}$, the ${ }^{6} \mathrm{Li} /{ }^{7} \mathrm{Li}$ ratio is less than $3 \times 10^{-5}$ and much smaller than the solar-system value (about $8.1 \times 10^{-2}$; Anders and Grevesse, 1989). The same things can be said in the case of ${ }^{9} \mathrm{Be}:{ }^{9} \mathrm{Be} /{ }^{16} \mathrm{O}$ is equal to or smaller than $2 \times 10^{-7}$ and the ${ }^{9} \mathrm{Be} /{ }^{7} \mathrm{Li}$ ratio is less than $2 \times 10^{-4}$. Although the abundance ratio of ${ }^{9} \mathrm{Be}$ to ${ }^{16} \mathrm{O}$ (if we adopt the maximum value) is almost comparable to the solarsystem value, ${ }^{9} \mathrm{Be} /{ }^{7} \mathrm{Li}$ is far from the solar-system value by two orders of magnitude or more.

The above results, which are obtained from a simulation of the standard model, seems to depend on the adopted models of the neutrino emission as well as the supernova explosion. However, we will see in the next section and in the subsequent papers that these results do not depend on the adopted model and, hence, ${ }^{6} \mathrm{Li}$ and ${ }^{9} \mathrm{Be}$ to ${ }^{7} \mathrm{Li}$ ratios as well as ${ }^{6} \mathrm{Li}$ and ${ }^{9} \mathrm{Be}$ to ${ }^{16} \mathrm{O}$ ratios are important measures for finding presolar grains of supernova origin.

\subsection{Production of $\mathrm{C}$-, $\mathrm{N}$-, and $\mathrm{O}$-isotopes}

Almost all of C-, N-, and O-isotopes are not produced by an appreciable amount (say, compared with the solar-system abundances) in the He-layer nor in the H-rich envelope as seen from Fig. 9. Especially, the abundances of ${ }^{12} \mathrm{C}$ and ${ }^{16} \mathrm{O}$ (also ${ }^{18} \mathrm{O}$ ) do not change from their initial abundances. Isotopes traced by the horizontal lines in Fig. 9 keep their initial abundances without addition and annihilation due to nuclear processes (see also Fig. 4). Key reactions relevant to the formation of these isotopes are summarized in Table 3. Only in the convective region of the He-layer $\left(M_{r} \lesssim 6.0 M_{\odot}\right)$, a small amount of ${ }^{13} \mathrm{C}$ is synthesized through mainly ${ }^{12} \mathrm{C}(n, \gamma){ }^{13} \mathrm{C}$ and ${ }^{12} \mathrm{C}(p, \gamma){ }^{13} \mathrm{~N}(n, p){ }^{13} \mathrm{C}$ where protons and neutrons are supplied from ${ }^{4} \mathrm{He}\left(v, v^{\prime} p\right)^{3} \mathrm{H}$ and ${ }^{4} \mathrm{He}\left(v, v^{\prime} n\right)^{3} \mathrm{He}$. In the innermost region, a part of synthesized ${ }^{13} \mathrm{C}$ is decomposed by ${ }^{13} \mathrm{C}(\alpha, n){ }^{16} \mathrm{O}$ after the shock passage and the resultant abundance of ${ }^{13} \mathrm{C}$ remains at relatively low level (about $1 \times 10^{-6}$ by mass fraction). It is conjectured that the amount of synthesized ${ }^{13} \mathrm{C}$ depends on the initial abundance of ${ }^{12} \mathrm{C}$. However, the ratio ${ }^{12} \mathrm{C} /{ }^{13} \mathrm{C}$, which will be shown in Fig. 13, would be almost independent of the initial abundance of ${ }^{12} \mathrm{C}$ since only ${ }^{12} \mathrm{C}$ is a parent isotope of ${ }^{13} \mathrm{C}$.

In the inner region of the He-layer, $\left(M_{r} \lesssim 5.0 M_{\odot}\right) \mathrm{N}$ isotopes are also produced. Almost all of ${ }^{14} \mathrm{~N}$ comes from the decomposition product of ${ }^{11} \mathrm{~B}$, i.e., ${ }^{11} \mathrm{~B}(\alpha, n){ }^{14} \mathrm{~N}$, in the shock-induced burning stage. The final abundance of ${ }^{14} \mathrm{~N}$ increases with an increase in the shock temperature (i.e., with a decrease in radius): it is of the order of $4 \times 10^{-6}$ (by mass fraction) in the innermost region $\left(M_{r} \lesssim 4.0 M_{\odot}\right)$. When $M_{r} \gtrsim 5.0 M_{\odot}$, it becomes below the level of its initial abundance (about $6 \times 10^{-8}$ ). Heavy isotope, ${ }^{15} \mathrm{~N}$, is synthesized through ${ }^{18} \mathrm{O}(p, \alpha){ }^{15} \mathrm{~N}$ in the preshock stage, corresponding to the hot CNO-cycle (protons are formed by the $v$-process, $\left.{ }^{4} \mathrm{He}\left(v, v^{\prime} p\right)^{3} \mathrm{H}\right)$. However, the amount of ${ }^{15} \mathrm{~N}$ is relatively low, $7 \times 10^{-7}$ (the innermost region) to $2 \times 10^{-8}$ (the outer region) by mass fraction. Among O-isotopes, only ${ }^{17} \mathrm{O}$ is synthesized in the convective He-layer, through ${ }^{16} \mathrm{O}(n, \gamma){ }^{17} \mathrm{O}$ in the preshock and the shock-induced burning stages. As in the case of ${ }^{15} \mathrm{~N}$, the abundance level is very low and the ratio, ${ }^{16} \mathrm{O} /{ }^{17} \mathrm{O}$, is as large as $1 \times 10^{4}$ or $5 \times 10^{4}$ except the innermost region $\left(M_{r} \lesssim 4.0 M_{\odot}\right)$ where the ratio is comparable to the solar-system ratio, $1 \times 10^{3}$.

In the radiative $\mathrm{He}$-layer all $\mathrm{CNO}$-elements keep their initial amounts. In this region protons are not much enough to change the initial abundances of the CNO-elements in a small time interval during the explosion. Productions of ${ }^{13} \mathrm{C}$ and ${ }^{17} \mathrm{O}$ through $n$-captures do not proceed effectively in the radiative He-layer because the initial amounts of ${ }^{12} \mathrm{C}$ and ${ }^{16} \mathrm{O}$ are less than those in the convective $\mathrm{He}-$ layer and because the amount of neutron is less than onetenth as little as that in the convective He-layer. In the deep region of the H-rich envelope, only ${ }^{15} \mathrm{~N}$ and ${ }^{18} \mathrm{O}$ change from their initial values. During the explosion ${ }^{15} \mathrm{~N}$ is produced through ${ }^{14} \mathrm{~N}(p, \gamma){ }^{15} \mathrm{O}\left(\beta^{+}\right){ }^{15} \mathrm{~N}$ and ${ }^{18} \mathrm{O}$ is decomposed through ${ }^{18} \mathrm{O}(p, \alpha){ }^{15} \mathrm{~N}$. In the region where $M_{r} \gtrsim 7.6 M_{\odot}$, all $\mathrm{CNO}$-elements keep their initial compositions of presupernova stage because of the shock temperature is too low to proceed nuclear reactions appreciably.

We briefly compare our result with those in Woosley et al. (1990) and a $25 M_{\odot}$ supernova model in Woosley and Weaver (1995). The main difference from the result of Woosley et al. (1990) is the amount of ${ }^{17} \mathrm{O}$. This is because, as seen 
in Section 2, we adopted the reaction rate of ${ }^{17} \mathrm{O}(p, \alpha){ }^{14} \mathrm{~N}$ from Landré et al. (1990), which is about six times larger than that in Caughlan and Fowler (1988). The difference of the amounts of the other CNO-elements between this study and Woosley et al. (1990) is roughly within one order of magnitude or so. In the convective He-layer, the amount of ${ }^{18} \mathrm{O}$ is different by a factor of 1000 . This is also due to the fact that, as mentioned in Section 2, the reaction rate of ${ }^{18} \mathrm{O}(\alpha, \gamma){ }^{22} \mathrm{Ne}$ becomes large by a factor of 10 to 100 in the temperature range of the He-layer after Caughlan and Fowler (1988).

\section{Influence of the Adopted Neutrino Emission Model}

Since light elements are produced mainly through neutrino interactions with helium and carbon as seen in the previous section, we expect that the production of light elements is influenced essentially by the adopted model of the neutrino emission during the supernova explosion. So uncertainties of the neutrino emission model give a confusion on studying the nucleosynthesis of light elements. To examine the influence and to find a suitable manner to discuss freely from the uncertainties of the neutrino emission model, we calculate the abundances of light elements changing the two parameters, namely, the decay time of the neutrino flux, $\tau_{\nu}$, and the total energy carried by neutrinos, $E_{v}$ as mentioned in Subsection 3.3.

\subsection{Abundance response to $\tau_{v}$ and $E_{v}$}

Roughly speaking, the abundance of a light element is determined by three kinds of processes, i.e., synthesis due to the $v$-process in the preshock stage, rapid production and decomposition at the stage of the shock heating, and re-synthesis in the shock-induced burning stage. If $\tau_{v}$ is much shorter than the arrival time of the shock, too small number of neutrinos remain at the stage of re-synthesis. In this case the final abundances of light elements are almost determined by the decomposition due to the shock heating. Because the inner region of the He-layer is exposed to the higher shock temperature, light elements survive in the outer region more than in the inner region. On the other hand, in the case where $\tau_{v}$ is much longer than the shock arrival time, enough number of neutrinos take part in the re-synthesis of light elements even after the decomposition due to the shock heating and the loss is recovered.

Another parameter, $E_{v}$, also affects the production rates of light elements. In our present study, $E_{v}$ means directly the total number flux of emitted neutrinos because we assume that, as mentioned in Subsection 3.3 (see Eq.(17)), the neutrino temperatures are fixed. So it may be conjectured that the amounts of synthesized light elements increase proportionally with $E_{v}$. However, the $v$-processes also produce, sometimes, "poison" to some kind of elements, so that the final abundances response to the adopted value of $E_{v}$ complicatedly. In this subsection we will see, as an example, how $\tau_{v}$ and $E_{v}$ influence the final abundance of ${ }^{11} \mathrm{~B}$.

In Fig. 10(a), we present the amount of ${ }^{11} \mathrm{~B}$ produced in the He-layer in the three cases with different $\tau_{\nu}$, i.e., $1 \mathrm{~s}, 3 \mathrm{~s}$, and $10 \mathrm{~s}\left(E_{v}\right.$ is fixed to be $\left.3 \times 10^{53} \mathrm{erg}\right)$. In the outer region where $M_{r} \gtrsim 5.0 M_{\odot}{ }^{11} \mathrm{~B}$ is mainly produced through ${ }^{12} \mathrm{C}\left(v, v^{\prime} p\right){ }^{11} \mathrm{~B}$ and the shock temperature is not high enough to decompose
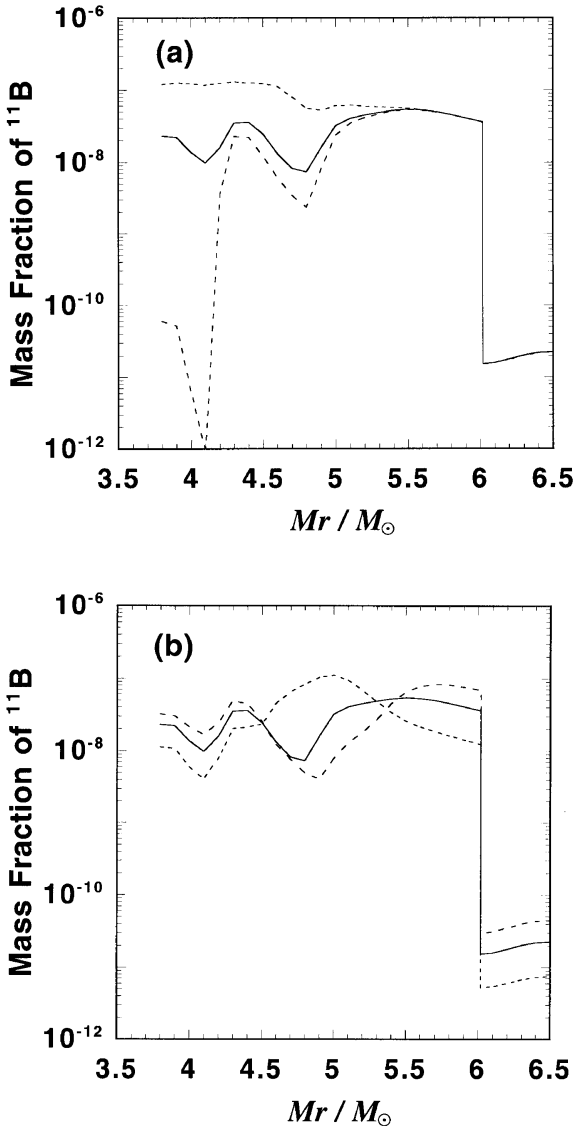

Fig. 10. Distribution of the final mass fraction of ${ }^{11} \mathrm{~B}$ in the He-layer. Panel (a) shows the cases of $\tau_{v}=1 \mathrm{~s}$ (dashed curve), $3 \mathrm{~s}$ (solid curve), and $10 \mathrm{~s}$ (dotted curve) with common $E_{v}\left(=3 \times 10^{53} \mathrm{erg}\right)$ and panel (b) the cases of $E_{v}=6 \times 10^{53} \mathrm{erg}$ (dashed curve), $3 \times 10^{53} \mathrm{erg}$ (solid curve), and $1 \times 10^{53} \mathrm{erg}$ (dotted curve) with common $\tau_{v}(=3 \mathrm{~s}$ ).

${ }^{11} \mathrm{~B}\left({ }^{11} \mathrm{~B}\right.$ is decomposed above $\left.2.5 \times 10^{8} \mathrm{~K}\right)$. As a result, the abundance of ${ }^{11} \mathrm{~B}$ does not depend appreciably on $\tau_{v}$. In the radiative He-layer where $M_{r} \gtrsim 6.0 M_{\odot}$, the ${ }^{11} \mathrm{~B}$ abundance is much less than that in the inner convective He-layer. This is due to the fact that there exists a small number of ${ }^{12} \mathrm{C}$ in the radiative He-layer compared with that in the inner convective He-layer. In the inner region where $M_{r} \lesssim 5.0 M_{\odot},{ }^{11} \mathrm{~B}$ is produced through ${ }^{12} \mathrm{C}\left(v, v^{\prime} n\right){ }^{11} \mathrm{C}\left(, e^{+} v_{e}\right){ }^{11} \mathrm{~B}$ and ${ }^{4} \mathrm{He}\left(v, v^{\prime} n\right)$ ${ }^{3} \mathrm{He}(\alpha, \gamma){ }^{7} \mathrm{Be}(\alpha, \gamma){ }^{11} \mathrm{C}\left(, e^{+} v_{e}\right){ }^{11} \mathrm{~B}$. This region is characterized by the high shock temperature; ${ }^{11} \mathrm{~B}$ produced through the $\nu$-process is decomposed almost completely through $\alpha$ - and $p$-capture reactions and, thus, the final abundance depends on the total neutrino flux as well as the shock temperature. In the case where $\tau_{v}=10 \mathrm{~s}$, the loss due to decomposition is recovered almost completely through the thermonuclear reaction sequence in the postshock stage as in the case of the outermost region (the shock reaches a sphere of $M_{r}=5.0 M_{\odot}$ at $27.3 \mathrm{~s}$ ) and the abundance pattern becomes almost uniform independently of the mass coordinate, $M_{r}$. In the case of $\tau_{v}=3 \mathrm{~s},{ }^{11} \mathrm{~B}$ is decomposed owing to the shock heating but a part is re-produced. However, in the case of $\tau_{v}=1 \mathrm{~s}$ almost all of ${ }^{11} \mathrm{~B}$ is decomposed by the shock heating (especially, in the innermost region where $M_{r} \lesssim 4.2 M_{\odot}$ ) and the final abundance is about one to five orders of magnitude lower than that in the case of $\tau_{v}=10 \mathrm{~s}$. 

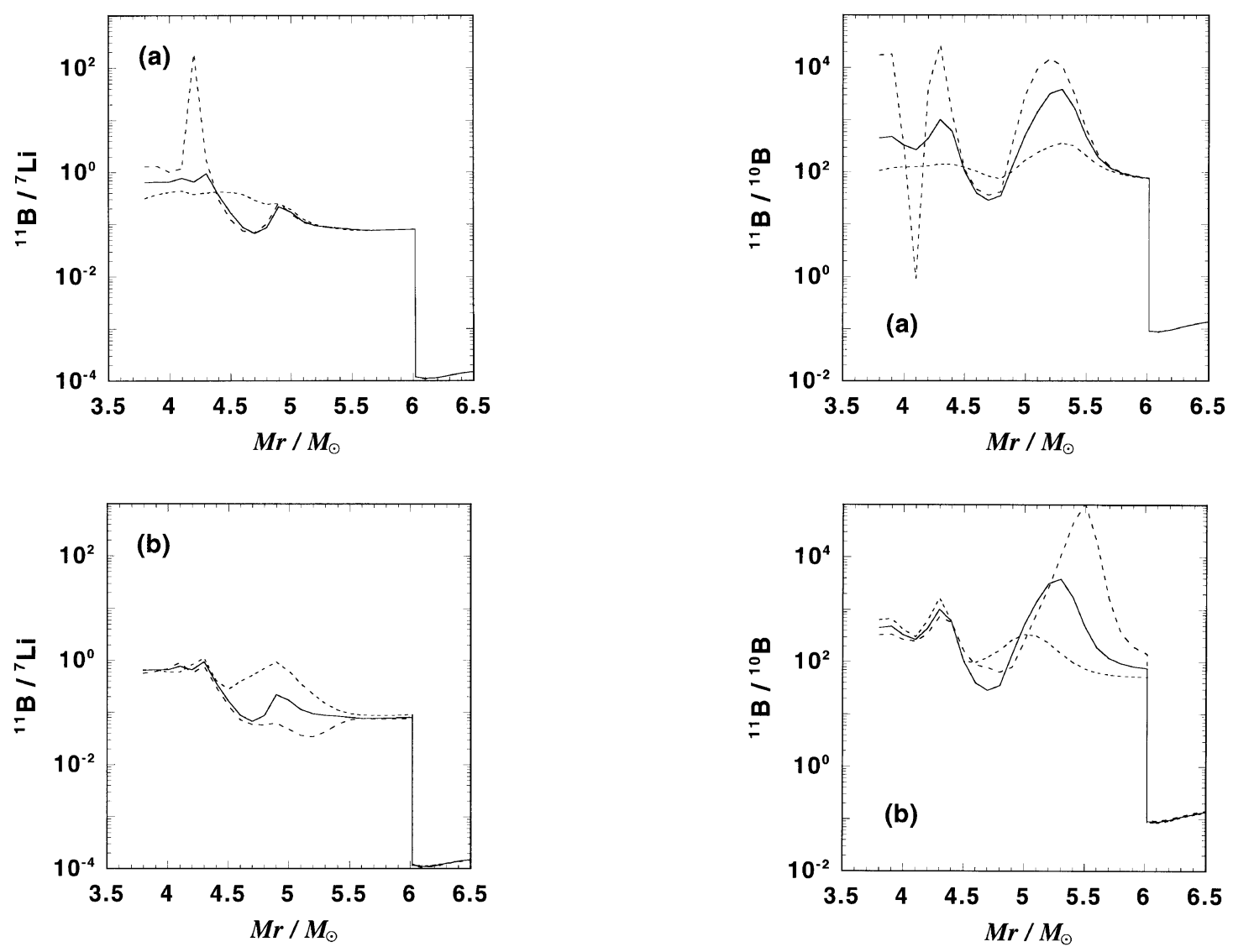

Fig. 11. Distribution of the number ratio, ${ }^{11} \mathrm{~B} /{ }^{7} \mathrm{Li}$ in the He-layer. Panels (a) and (b) are illustrated by the same manner as in Fig. 10.

Fig. 12. The same as Fig. 11 but for ${ }^{11} \mathrm{~B} /{ }^{10} \mathrm{~B}$.

In Fig. 10(b) the distribution curves of produced ${ }^{11} \mathrm{~B}$ are illustrated for the three cases with different $E_{v}$ (but $\tau_{v}$ is fixed to be $3 \mathrm{~s}$ ). As a whole, the distribution curves behave moderately in contract to the case of Fig. 10(a) and the variation of the amount is confined within an order of magnitude or so. As conjectured earlier, as long as we are concerned with the outermost region where $M_{r} \gtrsim 5.7 M_{\odot}$, the amount of ${ }^{11} \mathrm{~B}$ is almost proportional to $E_{\nu}$. But in the inner region, the distribution curves behave in a different manner from point to point. Especially, in the middle region where $4.5 M_{\odot} \lesssim M_{r} \lesssim 5.5 M_{\odot}$, the amount of ${ }^{11} \mathrm{~B}$ decreases with an increase in $E_{v}$. This is due to the fact that $(p, \alpha)$-reaction, by which ${ }^{11} \mathrm{~B}$ is decomposed (see Table 3 ), becomes inactive with an decrease in $E_{v}$ because protons are supplied mainly by ${ }^{4} \mathrm{He}\left(v, v^{\prime} p\right)^{3} \mathrm{H}$.

\subsection{Abundance ratios among the $X$-elements}

We can expect that, at least qualitatively, the $X$-elements are processed by a similar manner and that the abundance ratios among them are less sensitive to the adopted values of $\tau_{v}$ and $E_{v}$ than the amounts themselves. In Figs. 11(a) and $11(\mathrm{~b})$, the number ratios of ${ }^{11} \mathrm{~B} /{ }^{7} \mathrm{Li}$ are shown in the cases with various $\tau_{v}$ and $E_{v}$, respectively. Except a narrow region between $M_{r}=4.1 M_{\odot}$ and $4.3 M_{\odot},{ }^{11} \mathrm{~B} /{ }^{7} \mathrm{Li}$ has a value between $6 \times 10^{-2}$ and 2 in the convective He-layer; the ratio of the maximum to the minimum in the convective Helayer is much smaller than those of the abundance curves shown in Fig. 10(a). In the outer region where $M_{r} \gtrsim 5.0 M_{\odot}$ ${ }^{7} \mathrm{Li}$ as well as ${ }^{11} \mathrm{~B}$ are produced through the $v$-processes in the preshock stage and scarcely decomposed by the shock heating. So the amount of ${ }^{7} \mathrm{Li}$ as well as ${ }^{11} \mathrm{~B}$ (and, hence, their ratio) are independent of $\tau_{v}$. In the region between 4.3 $M_{\odot}$ and $5.0 M_{\odot},{ }^{11} \mathrm{~B} /{ }^{7} \mathrm{Li}$ ratios have almost the same values for the cases of $\tau_{v}=1 \mathrm{~s}$ and $3 \mathrm{~s}$ where most of neutrinos pass through before the shock passage. Both ${ }^{7} \mathrm{Li}$ and ${ }^{11} \mathrm{~B}$ are firstly produced through $v$-processes but decomposed almost completely during the shock-induced burning stage. In the case of $\tau_{v}=10 \mathrm{~s}$, only ${ }^{11} \mathrm{~B}$ is recovered almost completely and, as a result, ${ }^{11} \mathrm{~B} /{ }^{7} \mathrm{Li}$ becomes slightly large compared with the cases of $\tau_{\nu} \lesssim 3 \mathrm{~s}$. In the innermost region where $M_{r} \lesssim 4.1 M_{\odot}$, the amounts of ${ }^{7} \mathrm{Be}$ and ${ }^{11} \mathrm{C}$, which are parent nuclei of ${ }^{7} \mathrm{Li}$ and ${ }^{11} \mathrm{~B}$, respectively, are almost determined by a steady-flow equilibrium condition of ${ }^{4} \mathrm{He}$ even in the case of $\tau_{v}=1 \mathrm{~s}$ so that ${ }^{11} \mathrm{~B} /{ }^{7} \mathrm{Li}$ ratio scarcely depends on $\tau_{v}$.

In the case of $\tau_{v}=1 \mathrm{~s}$ the ${ }^{11} \mathrm{~B} /{ }^{7} \mathrm{Li}$ ratio becomes exceptionally large in the narrow region between $M_{r}=4.1 M_{\odot}$ and $4.3 M_{\odot}$. In this case, neutrino irradiation stops before the shock arrival and both ${ }^{7} \mathrm{Be}$ and ${ }^{11} \mathrm{C}$ decrease exponentially in the post shock stage. Since the decomposition rate of ${ }^{7} \mathrm{Be}$ is larger than that of ${ }^{11} \mathrm{C}$ and ${ }^{11} \mathrm{~B} /{ }^{7} \mathrm{Li}$ ratio becomes very large. As seen in Fig. $11(\mathrm{~b}),{ }^{11} \mathrm{~B} /{ }^{7} \mathrm{Li}$ varies moderately with $E_{v}$. In the inner $\left(M_{r} \lesssim 4.3 M_{\odot}\right)$ and the outer $\left(M_{r} \gtrsim 5.5 M_{\odot}\right)$ regions, the ratio does not depend on $E_{v}$. In the middle region the ratio increases with a decrease in $E_{\nu}$ because of ${ }^{11} \mathrm{~B}$ behavior mentioned in the last paragraph.

Compared with ${ }^{11} \mathrm{~B} /{ }^{7} \mathrm{Li}$ ratio, ${ }^{11} \mathrm{~B} /{ }^{10} \mathrm{~B}$ ratio behaves in a different way against $\tau_{\nu}$. We see from Fig. $12\left(\right.$ a) that ${ }^{11} \mathrm{~B} /{ }^{10} \mathrm{~B}$ 


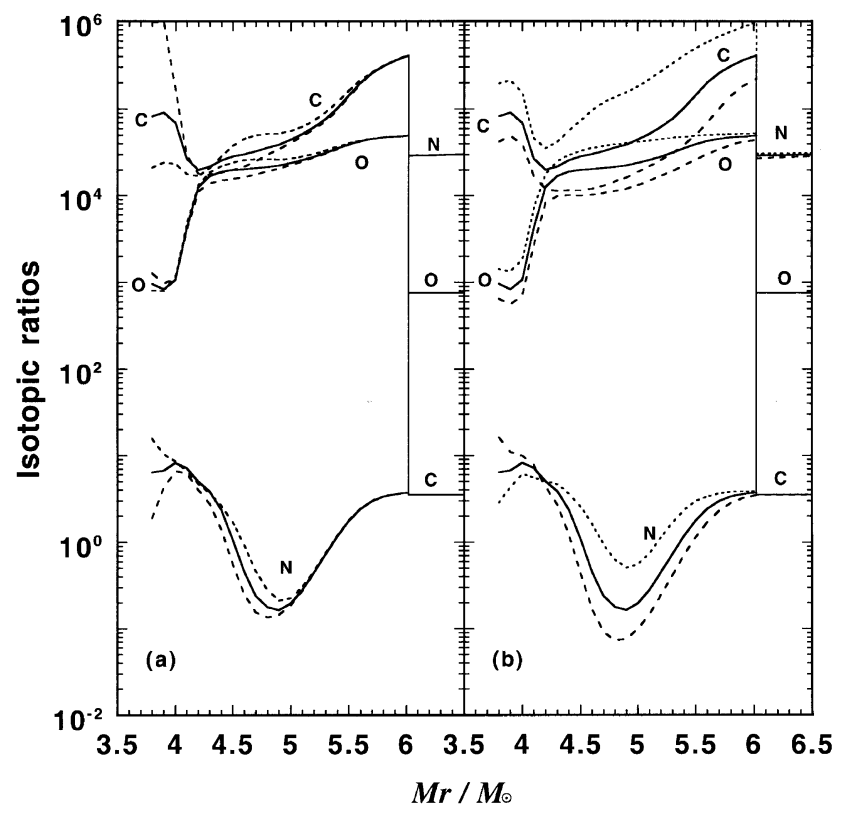

Fig. 13. The same as Fig. 11 but in the cases of ${ }^{12} \mathrm{C} /{ }^{13} \mathrm{C},{ }^{14} \mathrm{~N} /{ }^{15} \mathrm{~N}$, and ${ }^{16} \mathrm{O} /{ }^{17} \mathrm{O}$, the curves of which are denoted by $\mathrm{C}, \mathrm{N}$, and $\mathrm{O}$, respectively.

ratio depends sensitively on $\tau_{\nu}$, especially, in the inner region where $M_{r} \lesssim 5.3 M_{\odot}$. This is due, mainly, to the fact that the amount of ${ }^{10} \mathrm{~B}$ is influenced strongly by the adopted value of $\tau_{v}$. In the He-layer, ${ }^{10} \mathrm{~B}$ is commonly synthesized through ${ }^{12} \mathrm{C}\left(v, v^{\prime} n p\right){ }^{10} \mathrm{~B}$. On the other hand, ${ }^{10} \mathrm{~B}$ is decomposed by $(p, \alpha)-,(n, \alpha)-$, and $(\alpha, p)$-reactions; in the innermost Helayer $\left(M_{r} \lesssim 4.5 M_{\odot}\right)(\alpha, p)$-reactions dominates and in the radiative He-layer $\left(M_{r} \gtrsim 6.0 M_{\odot}\right)(p, \alpha)$-reaction does. Furthermore, it should be noted that protons are supplied by the $v$-process ${ }^{4} \mathrm{He}\left(v, v^{\prime} p\right)^{3} \mathrm{H}$ and neutrons are formed mainly by ${ }^{4} \mathrm{He}\left(v, v^{\prime} n\right)^{3} \mathrm{He}$ and ${ }^{3} \mathrm{H}\left({ }^{3} \mathrm{H}, n n\right)^{4} \mathrm{He}$ in the preshock stage. Thus, the final abundance of ${ }^{10} \mathrm{~B}$ varies in a complicated manner depending on $\tau_{\nu}$ as well as the mass coordinates, $M_{r}$. The ratio of ${ }^{11} \mathrm{~B} /{ }^{10} \mathrm{~B}$, as shown in Figs. $12(\mathrm{~b})$, does not depend strongly on the adopted value of $E_{v}$ in the inner region where $M_{r} \lesssim 5.0 M_{\odot}$. However, in the outer region it scatters widely: from Fig. 12(b) we observe a difference of two or more orders of magnitude between cases with small and large $E_{v}$.

Thus, we can say that the abundance ratio ${ }^{11} \mathrm{~B} /{ }^{7} \mathrm{Li}$ is less sensitive to uncertainties on the neutrino emission model than other quantities such as ${ }^{11} \mathrm{~B} /{ }^{10} \mathrm{~B}$ and the amounts of $X$ elements themselves. Nevertheless, it should be noted that there remains a difference more than one order of magnitude in ${ }^{11} \mathrm{~B} /{ }^{7} \mathrm{Li}$ among the cases with various $\tau_{v}$ and $E_{v}$.

\subsection{Influence on ${ }^{6} \mathrm{Li}$ and ${ }^{9} \mathrm{Be}$}

Only in the inner region $\left(M_{r} \lesssim 5.0 M_{\odot}\right)$ of the convective He-layer where the shock wave passes through within a first $30 \mathrm{~s}$ after the beginning of the explosion, the amounts of produced ${ }^{6} \mathrm{Li}$ and ${ }^{9} \mathrm{Be}$ are affected by the value of $\tau_{v}$. Especially, in the case of $\tau_{v}=10 \mathrm{~s}$ the final abundances become large by two or three orders of magnitude compared with the standard case $\left(\tau_{v}=3 \mathrm{~s}\right)$ since the neutrino irradiation lasts even after the passage of the shock. However, the influence of the neutrino irradiation time on the amounts of ${ }^{6} \mathrm{Li}$ and ${ }^{9} \mathrm{Be}$ is of no importance since the abundance level is still below the detection limit. In the outer region where $M_{r} \gtrsim 5.0 M_{\odot}$, the amounts of ${ }^{6} \mathrm{Li}$ and ${ }^{9} \mathrm{Be}$ do not depend so much on $\tau_{v}$ as long as we are concerned with the cases where $\tau_{v} \lesssim 10 \mathrm{~s}$.

Roughly speaking, on the other hand, ${ }^{6} \mathrm{Li}$ and ${ }^{9} \mathrm{Be}$ are produced in proportion to the adopted value of $E_{v}$ (i.e., to the total number of neutrinos) since they are formed commonly through the $v$-processes with the target nuclei of ${ }^{12} \mathrm{C}$ and ${ }^{13} \mathrm{C}$. For example, when $E_{v}=6 \times 10^{53} \mathrm{erg}$ their maximum amounts (which are realized near $M_{r}=5.5 M_{\odot}$ ) become two times larger than those in the standard case $\left(E_{v}=3 \times 10^{53}\right.$ erg). However, the amount of ${ }^{7} \mathrm{Li}$ increases similarly and, as a result, both ${ }^{6} \mathrm{Li} /{ }^{7} \mathrm{Li}$ and ${ }^{9} \mathrm{Be} /{ }^{7} \mathrm{Li}$ are still less than $2 \times 10^{-4}$. Thus, the conclusions mentioned in Subsection 4.2 hold as they are irrelevant to the adopted model of neutrino emission.

\subsection{Influence on the CNO-elements}

In Fig. 13 we show how the isotopic ratios of CNO-elements depend on $\tau_{v}$ and $E_{v}$. In most part of the He-layer where $M_{r} \gtrsim 4.0 M_{\odot}$, isotopic ratios are very insensitive to the adopted value of $\tau_{v}$; all of their variations are confined within a factor of 4 . The CNO-elements (except ${ }^{12} \mathrm{C}$ and ${ }^{16} \mathrm{O}$ ) are produced by various kind of the $\nu$-processes whereas they are hardly decomposed by the shock heating, after they are once produced. As a result, the final abundances of these elements are governed entirely by the total number of neutrinos but not by the time variation of the neutrino flux.

Only in the innermost region $\left(M_{r} \lesssim 4.0 M_{\odot}\right)$ where the shock temperature is relatively high, the isotopic ratios depend weakly on $\tau_{v}$. The ratio ${ }^{12} \mathrm{C} /{ }^{13} \mathrm{C}\left(\right.$ or $\left.{ }^{14} \mathrm{~N} /{ }^{15} \mathrm{~N}\right)$ decreases (or increases) with an increase in $\tau_{v}$. As described in Subsection $4.3,{ }^{13} \mathrm{C}$ is decomposed through ${ }^{13} \mathrm{C}(\alpha, n){ }^{16} \mathrm{O}$ owing to the shock heating. After the shock passage ${ }^{13} \mathrm{C}$ is reproduced through ${ }^{12} \mathrm{C}(n, \gamma){ }^{13} \mathrm{C}$; neutrons are supplied by the $\nu$-process originated by the remaining neutrino flux. Thus, the final amount of ${ }^{13} \mathrm{C}$ becomes large when $\tau_{v}$ is long. Even in the case of $\tau_{v}=1 \mathrm{~s}$, about one-thousandth of the total number of neutrinos pass through a sphere of $M_{r}=3.8 M_{\odot}$ after the shock arrival. 
The ratio ${ }^{14} \mathrm{~N} /{ }^{15} \mathrm{~N}$ behaves inversely against $\tau_{\nu}$ compared to the case of ${ }^{12} \mathrm{C} /{ }^{13} \mathrm{C}$. Owing to the shock heating, ${ }^{14} \mathrm{~N}$ and ${ }^{15} \mathrm{~N}$ are both decomposed through $(\alpha, \gamma)$ - and $(p, \alpha)$ reaction, respectively. When $\tau_{v}$ is long, ${ }^{14} \mathrm{~N}$ is reproduced from ${ }^{11} \mathrm{~B}$, which is formed through a reaction sequence of $\alpha$ captures starting from ${ }^{4} \mathrm{He}\left(v, v^{\prime} p\right)^{3} \mathrm{H}$ after the shock passage. On the contrary, when $\tau_{v}$ is short, ${ }^{14} \mathrm{~N}$ is not reproduced and ${ }^{15} \mathrm{~N}$ is hardly decomposed because of lack of protons. Hence, ${ }^{14} \mathrm{~N} /{ }^{15} \mathrm{~N}$ becomes small compared to the case of long $\tau_{\nu}$. In the case of oxygen, ${ }^{17} \mathrm{O}$, once formed, is hardly decomposed by the shock heating even in the innermost region and, hence, the variation of ${ }^{16} \mathrm{O} /{ }^{17} \mathrm{O}$ is very small (within a factor of 2).

As conjectured earlier and seen from Fig. 13, the final amount of ${ }^{13} \mathrm{C}$ increases, more or less, in proportion to $E_{v}$, i.e., the total number of neutrinos, irrelevant to the internal mass coordinate $M_{r}$. As shown in Table $3,{ }^{13} \mathrm{C}$ is produced through the $v$-process due to ${ }^{12} \mathrm{C}$ and hardly decomposed by the shock heating. The ratio ${ }^{16} \mathrm{O} /{ }^{17} \mathrm{O}$ changes with $E_{v}$ similarly to the case of ${ }^{12} \mathrm{C} /{ }^{13} \mathrm{C}$. However, ${ }^{14} \mathrm{~N} /{ }^{15} \mathrm{~N}$ behaves differently against $E_{v}$. In the outer region where $M_{r} \gtrsim 4.3 M_{\odot}$, ${ }^{15} \mathrm{~N}$, which is mainly produced through ${ }^{18} \mathrm{O}(p, \alpha){ }^{15} \mathrm{~N}$ and ${ }^{16} \mathrm{O}\left(v, v^{\prime} p\right){ }^{15} \mathrm{~N}$, is increased with $E_{v}$. The amount of ${ }^{14} \mathrm{~N}$, however, scarcely depends on $E_{v}$ because the amount of ${ }^{11} \mathrm{~B}$, parent nucleus of ${ }^{14} \mathrm{~N}$, is determined by a balance between production and decomposition reaction, both of which are originated by the $\nu$-processes. Thus, ${ }^{14} \mathrm{~N} /{ }^{15} \mathrm{~N}$ becomes low when $E_{v}$ is large. In the inner region, however, ${ }^{14} \mathrm{~N} /{ }^{15} \mathrm{~N}$ ratio increases with an increase in $E_{\nu}$ because the amount of ${ }^{14} \mathrm{~N}$ increases and that of ${ }^{15} \mathrm{~N}$ decreases. The reason is as follows. As seen in Table $3,{ }^{14} \mathrm{~N}$ is produced through an $\alpha$-capture reaction sequence originated by the $v$-process. Increase in $E_{v}$ brings about the increment of ${ }^{14} \mathrm{~N}$ production and, simultaneously, the same reaction provides more protons which are the poison of ${ }^{15} \mathrm{~N}$.

\section{Conclusions and Discussion}

In order to find a suitable manner for distinguishing presolar grains of supernova origin from those of the other origins, we investigated the light element synthesis in the He-layer and the H-rich envelope of a $16.2 M_{\odot}$ supernova and calculated their final abundances as well as the abundance ratios by the use of our nuclear reaction network. The abundances of light elements are affected by various kinds of physical parameters which govern the models of the supernova explosion as well as the neutrino emission. Of these parameters, the decay time of the neutrino flux, $\tau_{v}$, and the total energy carried by neutrinos, $E_{v}$, are most important since the explosive synthesis of light elements is initiated, in most cases, by the $v$-processes. In the present study we concentrated ourselves on investigating the influence of uncertainties in the adopted model of the neutrino emission on the abundances of light elements. The obtained results are summarized as follows:

1) The amounts of ${ }^{6} \mathrm{Li}$ and ${ }^{9} \mathrm{Be}$ produced in the exploding He-layer and the H-rich envelope are much smaller than that of, for example, ${ }^{7} \mathrm{Li}$. The ratios of ${ }^{6} \mathrm{Li} /{ }^{7} \mathrm{Li}$ and ${ }^{9} \mathrm{Be} /{ }^{7} \mathrm{Li}$ are less than $3 \times 10^{-5}$ and $2 \times 10^{-4}$, respectively, as long as we are concerned with the case where the isotopic abundance exceeds beyond the detection limit (i.e., the number ratio to ${ }^{16} \mathrm{O}$ is greater than $1 \times 10^{-9}$ ).
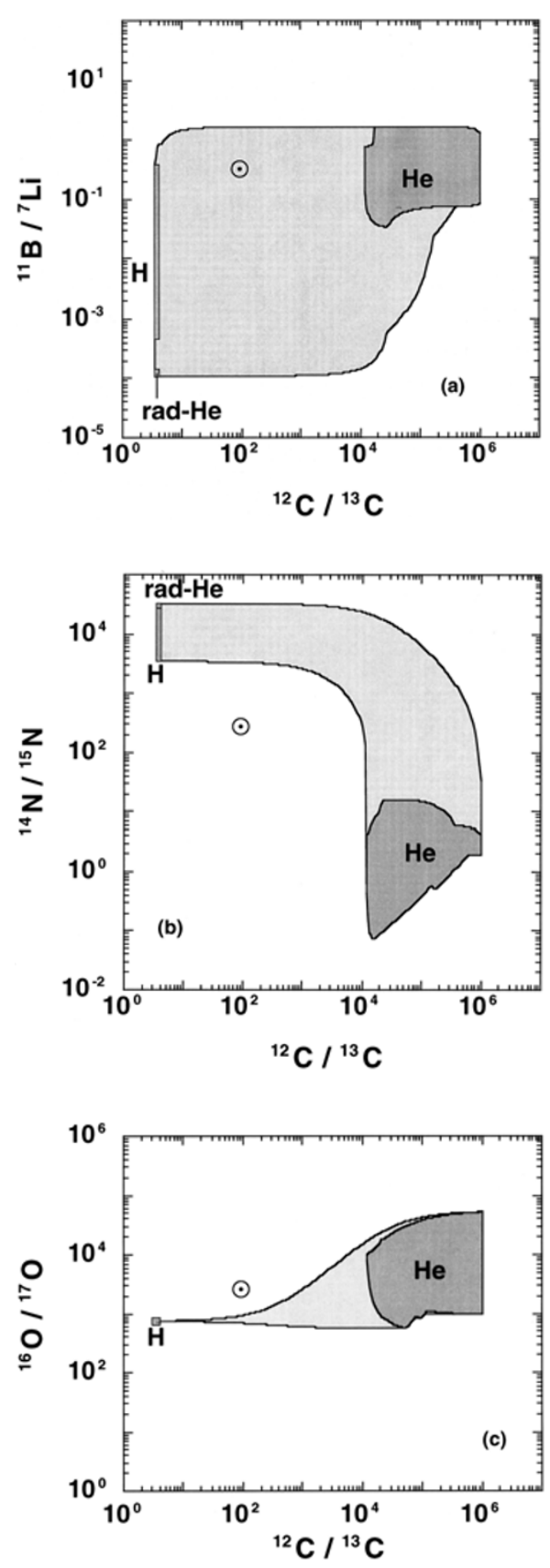

Fig. 14. The number ratios of ${ }^{11} \mathrm{~B} /{ }^{7} \mathrm{Li}(\operatorname{panel}(\mathrm{a})),{ }^{14} \mathrm{~N} /{ }^{15} \mathrm{~N}$ (panel (b)), and ${ }^{16} \mathrm{O} /{ }^{17} \mathrm{O}$ (panel (c)) versus ${ }^{12} \mathrm{C} /{ }^{13} \mathrm{C}$. The adopted values of $\tau_{v}$ and $E_{v}$ are those in Eqs. (18) and (19). Three regions with a dark shade are of the convective He-layer (denoted by $\mathrm{He}$ ), the radiative He-layer (denoted by rad-He), and the $\mathrm{H}$-rich envelope (denoted by $\mathrm{H}$ ) whereas a region with a light shade denotes the ratios made of the mixing between these regions. In panel (c), the region of the radiative He-layer overlaps with that of the H-rich envelope. A mark $\odot$ shows the ratio of the solar-system composition. (In panel (a), exceptional spike appeared in Fig. 11(a) is excluded.)

The above ratios of ${ }^{6} \mathrm{Li} /{ }^{7} \mathrm{Li}$ and ${ }^{9} \mathrm{Be} /{ }^{7} \mathrm{Li}$ are far from the corresponding solar-system values.

2) Among $X$-elements, ${ }^{7} \mathrm{Li},{ }^{10} \mathrm{~B}$, and ${ }^{11} \mathrm{~B}$ are synthesized appreciably (i.e., beyond the level of the detection limit) in the He-layer and the inner H-rich envelope of a supernova. The abundances of these isotopes vary over several orders of magnitude depending on the internal 
mass coordinate, $M_{r}$, as well as the neutrino emission model (i.e., $\tau_{v}$ and $E_{v}$ ). However, the ratio of ${ }^{11} \mathrm{~B} /{ }^{7} \mathrm{Li}$ is confined within a relatively small region (but, ${ }^{11} \mathrm{~B} /{ }^{10} \mathrm{~B}$ or ${ }^{10} \mathrm{~B} /{ }^{7} \mathrm{Li}$ scatters widely as the abundances do).

3) The tightly bound isotopes, ${ }^{12} \mathrm{C}$ and ${ }^{16} \mathrm{O}$, are never synthesized in the He-layer nor in the H-rich envelope and the abundances are completely determined by their initial values. Heavy isotope, ${ }^{18} \mathrm{O}$, keeps, in most case, its initial abundance.

4) The other CNO-elements, ${ }^{13} \mathrm{C},{ }^{14} \mathrm{~N},{ }^{15} \mathrm{~N}$, and ${ }^{17} \mathrm{O}$ are synthesized in the convective He-layer by small fractions (but not outside the radiative He-layer). The amounts of these elements vary with the internal mass coordinate, $M_{r}$, by two or more orders of magnitude. They also depend on $\tau_{v}$ and $E_{v}$ but are confined within a factor of 10 or so if we observe at fixed $M_{r}$.

As pointed out in Section 1, several procedures for distinguishing presolar grains of supernova origin have been proposed (e.g, Nittler et al., 1996; Travaglio et al., 1999). So, it may be worthwhile to compile our numerical results for applying to the study of presolar grains of supernova origin. According to familiar ways of cosmochemists, we make useful three diagrams between two isotopic (elemental) ratios overwriting all of numerical results presented in this study: diagrams of ${ }^{11} \mathrm{~B} /{ }^{7} \mathrm{Li}-{ }^{12} \mathrm{C} /{ }^{13} \mathrm{C},{ }^{14} \mathrm{~N} /{ }^{15} \mathrm{~N}-{ }^{12} \mathrm{C} /{ }^{13} \mathrm{C}$, and ${ }^{16} \mathrm{O} /{ }^{17} \mathrm{O}-{ }^{12} \mathrm{C} /{ }^{13} \mathrm{C}$, which are illustrated in Figs. 14(a) to 14(c). The adopted values of $\tau_{v}$ and $E_{v}$ are those in Eqs. (18) and (19). As described in the last section, these ratios vary both with the adopted values of $\tau_{v}$ and $E_{v}$ and with the internal mass coordinate we observe. Nevertheless, they are confined within relatively small, two regions in the diagram: one is of the H-rich envelope (including the radiative Helayer) and another is of the inner convective He-layer. The corresponding solar-system value (marked by $\odot$ in the figures) does not fall onto the two regions mentioned above. This would help us to distinguish presolar grains of supernova origin from solar-system materials.

It should be noted here that there happens to occur a material mixing among the exploding layers, which is caused by the Rayleigh-Taylor instability (e.g., Hachisu et al., 1994). The mixing disturbs inevitably the isotopic (elemental) ratios of materials. When there occurs a mixing between two arbitrary fluid elements outside the He-burning shell (i.e., the He-layer and the H-rich envelope), we can readily evaluate the mixing effect on the isotopic ratios using our present results. The variation of the isotopic ratios due to the material mixing are shown in Figs. 14(a) to 14(c) by light shadows. Thus, we propose that dust-grains originated from a $16.2 M_{\odot}$ supernova should have the following cosmochemical features of isotopic (elemental) ratios of the light elements:

(a) Among the $X$-elements, ${ }^{6} \mathrm{Li}$ and ${ }^{9} \mathrm{Be}$ should be both very rare. Presolar grains with ${ }^{6} \mathrm{Li} /{ }^{7} \mathrm{Li}$ less than $3 \times 10^{-5}$ and with ${ }^{9} \mathrm{Be} /{ }^{7} \mathrm{Li}$ less than $2 \times 10^{-4}$ should be originated from the supernova.

(b) Presolar grains with the ratios ${ }^{11} \mathrm{~B} /{ }^{7} \mathrm{Li},{ }^{12} \mathrm{C} /{ }^{13} \mathrm{C},{ }^{14} \mathrm{~N} /{ }^{15} \mathrm{~N}$, and ${ }^{16} \mathrm{O} /{ }^{17} \mathrm{O}$ confined within relatively narrow two regions with dark shade shown in Figs. 14(a) to 14(c) should be also originated from the supernova. If there occurs a mixing between the He-layer and the H-rich envelope, the ratio would fall onto the regions with light shade.

We have a brief comment on comparison of Fig. 14(b) with Fig. 8(a) in Travaglio et al. (1999) which shows ${ }^{14} \mathrm{~N} /{ }^{15} \mathrm{~N}$ ${ }^{12} \mathrm{C} /{ }^{13} \mathrm{C}$ ratios in the $\mathrm{He} / \mathrm{C}$ zone and the $\mathrm{He} / \mathrm{N}$ zone of a $20 M_{\odot}$ supernova model in Woosley and Weaver (1995). The ratios in the $\mathrm{He} / \mathrm{N}$ zone of their model is well identified with those in the radiative He-layer in our model and the range of the ratios in the $\mathrm{He} / \mathrm{C}$ zone in their model is inside that in the convective He-layer in our model. So, roughly speaking, we can say that our result is consistent with their result on ${ }^{14} \mathrm{~N} /{ }^{15} \mathrm{~N}-{ }^{12} \mathrm{C} /{ }^{13} \mathrm{C}$ ratios. The comparison between our results and the ratios of low-density presolar graphite grains presented in Travaglio et al. (1999) brings us the same result as they obtained: the ratios of the all grains are outside the predicted range of the isotopic ratios. In addition to that, we also compared the range of ${ }^{16} \mathrm{O} /{ }^{17} \mathrm{O}-{ }^{12} \mathrm{C} /{ }^{13} \mathrm{C}$ ratios in Fig. $14(\mathrm{c})$ with the ratios of the presolar graphite grains in Travaglio et al. (1999) the ${ }^{16} \mathrm{O} /{ }^{17} \mathrm{O}$ ratios of which were measured. Most of the grains do not have the isotopic ratios predicted in Fig. 14(c). Consequently, the low-density graphite grains are not expected to be originated from the supernova $\left(16.2 M_{\odot}\right)$ even if the uncertainties on the $v$-processes are considered.

Of course, we know that the amounts of light elements synthesized in the supernova explosion are influenced by various kinds of physical processes which we do not take into account in our present study and that the above conclusions are not always in general. Among the ratios illustrated in Fig. 14, some are influenced by the adopted chemical compositions and almost determined by the initial ratios themselves (e.g., ${ }^{16} \mathrm{O} /{ }^{17} \mathrm{O}$ and ${ }^{12} \mathrm{C} /{ }^{13} \mathrm{C}$ in the H-rich envelope). In the above, we discussed the effect of a material mixing between fluid layers but it is not sufficient: Once there occurs a mixing, then not only the chemical compositions of the mixture change from those of pre-mixed layers but also the mixing would promote possibly a new kind of nuclear reactions through which the $X$-elements synthesized in the He-layer are decomposed by protons in the H-rich envelope. There may also occur an alternative mixing between the Helayer (or the H-rich envelope) and the deep layer such as the $\mathrm{C}$-layer and the $\mathrm{O} / \mathrm{Ne}$-layer. Furthermore, we have to consider the nucleosynthesis in supernovae with various masses up to, say, $25 M_{\odot}$.

In the second paper (Yoshida et al., 1999) we will investigate how the initial chemical compositions modify our present conclusions mentioned above and see that our present conclusions do not depends seriously on the initial conditions. In the third paper we will study the effects of the delayed nuclear reactions driven by the mixing as well as the mixing with deep layers. Moreover, using the supernova models with various masses we will discuss the light element synthesis in the subsequent paper. We will see from these papers that, although some are modified more or less, our present conclusions are almost general in the whole.

Acknowledgments. The authors would like to thank Kohichi Iwamoto, Ken'ichi Nomoto, and Toshikazu Shigeyama for giving the data of internal structure for a progenitor model, 14E1, and help- 
ful discussion. They are also indebted to Takafumi Hirata for useful advice from cosmochemical viewpoints. They also thank Shigeru Ida and Hidekazu Tanaka for useful advice and Kei Kudo for her help in preparing this manuscript. This work has been supported, in part, by Grand-in-Aid for General Scientific Research (B) (Nos. 07454114 and 09440089 ). The computation has been made by Cray C916 at the Computer Center of Tokyo Institute of Technology.

\section{References}

Amari, S., E. Anders, A. Virag, and E. Zinner, Interstellar graphite in meteorites, Nature, 345, 238-240, 1990.

Anders, E. and N. Grevesse, Abundances of the elements: Meteoritic and solar, Geochim. Cosmochim. Acta, 53, 197-214, 1989.

Arnett, W. D., Explosive nucleosynthesis in stars, Astrophys. J., 157, 1369$1380,1969$.

Arnett, W. D., J. N. Bahcall, R. P. Kirshner, and S. E. Woosley, Supernova 1987A, Annu. Rev. Astron. Astrophys., 27, 629-700, 1989.

Bahcall, J. N., Neutrino Astrophysics, pp. 428-432, Cambridge University Press, Cambridge, 1989.

Blackmon, J. C., A. E. Champagne, M. A. Hofstee, M. S. Smith, R. G. Downing, and G. P. Lamaze, Measurement of the ${ }^{17} \mathrm{O}(p, \alpha){ }^{14} \mathrm{~N}$ cross section at stellar energies, Phys. Rev. Lett., 74, 2642-2645, 1995.

Buchmann, L., New stellar reaction rate for ${ }^{12} \mathrm{C}(\alpha, \gamma){ }^{16} \mathrm{O}$, Astrophys. J., 468, L127-L130, 1996.

Buchmann, L., Errata, Astrophys. J., 479, L153, 1997.

Burrows, A. and J. M. Lattimer, Neutrinos from SN 1987A, Astrophys. J., 318, L63-L68, 1987.

Caughlan, G. R. and W. A. Fowler, Thermonuclear reaction rates V, Atom. Data Nucl. Data Tables, 40, 283-334, 1988.

Cooperstein, J., Neutrinos in supernovae, Phys. Rep., 163, 95-126, 1988.

Epstein, R. I., S. A. Colgate, and W. C. Haxton, Neutrino-induced $r$-process nucleosynthesis, Phys. Rev. Lett., 61, 2038-2041, 1988.

Hachisu, I., T. Matsuda, K. Nomoto, and T. Shigeyama, Mixing in ejecta of supernovae. II. Mixing width of 2D Rayleigh-Taylor instabilities in the helium star models for type Ib/Ic supernovae, Astron. Astrophys. Suppl., 104, 341-364, 1994.

Hoffman, R. D. and S. E. Woosley, Tables of reaction rates for nucleosynthesis for charged particle, weak, and neutrino interactions $(Z<45)$, (unpublished; http://isotopes.lbl.gov/isotopes/hw92_1.html), 1992.

Hoppe, P., S. Amari, E. Zinner, and R. S. Lewis, Isotopic compositions of C, $\mathrm{N}, \mathrm{O}, \mathrm{Mg}$, and $\mathrm{Si}$, trace element abundances, and morphologies of single circumstellar graphite grains in four density fractions from the Murchison meteorite, Geochim. Cosmochim. Acta, 59, 4029-4056, 1995.

Hutcheon, I. D., G. R. Huss, A. J. Fahey, and G. J. Wasserburg, Extreme ${ }^{26} \mathrm{Mg}$ and ${ }^{17} \mathrm{O}$ enrichments in an Orgueil corundum: identification of a presolar oxide grain, Astrophys. J., 425, L97-L100, 1994.

Iwamoto, K., T. R. Young, N. Nakasato, T. Shigeyama, K. Nomoto, I. Hachisu, and H. Saio, Instabilities and mixing in SN 1993J, Astrophys. J., 477, 865-875, 1997.

Käppeler, F., M. Wiescher, U. Giesen, J. Görres, I. Baraffe, M. El Eid, C. M Raiteri, M. Busso, R. Gallino, M. Limongi, and A. Chieffi, Reaction rates for ${ }^{18} \mathrm{O}(\alpha, \gamma)^{22} \mathrm{Ne},{ }^{22} \mathrm{Ne}(\alpha, \gamma)^{26} \mathrm{Mg}$, and ${ }^{22} \mathrm{Ne}(\alpha, n)^{25} \mathrm{Mg}$ in stellar helium burning and $s$-process nucleosynthesis in massive stars, Astrophys. J., 437, 396-409, 1994.

Lamb, S. A., I. Iben, Jr., and W. M. Howard, On the evolution of massive stars through the core carbon-burning phase, Astrophys. J., 207, 209-232, 1976.

Landré, V., N. Prantzos, P. Aguer, G. Bogaert, A. Lefebvre, and J. P. Thibaud, Revised reaction rates for the $\mathrm{H}$-burning of ${ }^{17} \mathrm{O}$ and the oxygen isotopic abundances in red giants, Astron. Astrophys, 240, 85-92, 1990.

Lattimer, J. M. and A. Yahil, Analysis of the neutrino events from supernova 1987A, Astrophys. J., 340, 426-434, 1989.

Mckeegan, K. D., R. M. Walker, and E. Zinner, Ion microprobe isotopic measurements of individual interplanetary dust particles, Geochim. Cosmochim. Acta, 49, 1971-1987, 1985.

Meyer, B. S., T. A. Weaver, and S. E. Woosley, Isotopic source table for a $25 M_{\odot}$ supernova, Meteoritics, 30, 325-334, 1995.
Myra, E. S. and A. Burrows, Neutrinos from type II supernovae: the first 100 milliseconds, Astrophys. J., 364, 222-231, 1990.

Nakamura, T., H. Umeda, K. Nomoto, F.-K. Thielemann, and A. Burrows, Nucleosynthesis in Type II supernovae and the abundances in metal-poor stars, Astrophys. J., 517, 193-208, 1999.

Nittler, L. R., S. Amari, E. Zinner, S. E. Woosley, and R. S. Lewis, Extinct ${ }^{44} \mathrm{Ti}$ in presolar graphite and $\mathrm{SiC}$ : proof of a supernova origin, Astrophys. J., 462, L31-34, 1996.

Nomoto, K. and M. Hashimoto, Presupernova evolution of massive stars, Phys. Rep., 163, 13-36, 1988.

Nomoto, K., F.-K. Thielemann, and K. Yokoi, Accreting white dwarf models for Type I supernovae. III. Carbon deflagration supernovae, Astrophys. J., 286, 644-658, 1984.

Rolfs, C. E. and W. S. Rodney, Cauldrons in the Cosmos, pp. 376-379, Chicago Press, Chicago and London, 1988

Saio, H., K. Nomoto, and M. Kato, Nitrogen and helium enhancement in the progenitor of supernova 1987A, Nature, 334, 508-510, 1988.

Sedov, L. I., Similarity and Dimensional Methods in Mechanics, pp. 260 270, Academic Press, New York and London, 1959.

Shigeyama, T. and K. Nomoto, Theoretical light curve of SN 1987A and mixing of hydrogen and nickel in the ejecta, Astrophys. J., 360, 242-256, 1990.

Shigeyama, T., K. Nomoto, M. Hashimoto, and D. Sugimoto, Light-curve models for supernova SN1987A in the Large Magellanic Cloud, Nature, 328, 320-323, 1987.

Spergel, D. N., T. Piran, A. Loeb, J. Goodman, and J. N. Bahcall, A simple model for neutrino cooling of the Large Magellanic Cloud Supernova, Science, 237, 1471-1473, 1987.

Swart, P. K., M. M. Grady, C. T. Pillinger, R. S. Lewis, and E. Anders, Interstellar carbon in meteorites, Science, 220, 406-410, 1983.

Thielemann, F.-K., M. Hashimoto, and K. Nomoto, Explosive nucleosynthesis in SN 1987A. II. Composition, radioactivities, and the neutron star mass, Astrophys. J., 349, 222-240, 1990

Thielemann, F.-K., K. Nomoto, and M. Hashimoto, Core-collapse supernovae and their ejecta, Astrophys. J., 460, 408-436, 1996.

Travaglio, C., R. Gallino, S. Amari, E. Zinner, S. E. Woosley, and R. S. Lewis, Low-density graphite grains and mixing in type II supernovae, Astrophys. J., 510, 325-354, 1999.

Weaver, T. A. and S. E. Woosley, Evolution and explosion of massive stars, Ann. New York Acad. Sci., 336, 335-357, 1980.

Wiescher, M., J. Görres, S. Graff, L. Buchmann, and F.-K. Thielemann, The hot proton-proton chains in low-metallicity objects, Astrophys. J., 343, 352-364, 1989.

Woosley, S. E., SN 1987A: After the peak, Astrophys. J., 330, 218-253, 1988.

Woosley, S. E. and T. A. Weaver, The physics of supernova explosions, Annu. Rev. Astron. Astrophys., 24, 205-253, 1986.

Woosley, S. E. and T. A. Weaver, The evolution and explosion of massive stars. II. Explosive hydrodynamics and nucleosynthesis, Astrophys. J. Suppl., 101, 181-235, 1995.

Woosley, S. E., D. H. Hartmann, R. D. Hoffman, and W. C. Haxton, The v-process, Astrophys. J., 356, 272-301, 1990.

Yoshida, T., Light elements synthesized in the He-layer and the H-rich envelope of type II supernovae, Doctoral Thesis, 2000.

Yoshida, T., K. Nakazawa, and H. Emori, Light elements synthesized in the He-layer and the H-rich envelope of a Type II supernova. II-Influence of initial chemical compositions-, Earth, Planets and Space, 1999 (submitted).

Zinner, E., Stellar nucleosynthesis and the isotopic composition of presolar grains from primitive meteorites, Annu. Rev. Earth Planet. Sci., 26, 147$188,1998$.

Zinner, E., M. Tang, and E. Anders, Interstellar SiC in the Murchison and Murray meteorites: Isotopic composition of $\mathrm{Ne}, \mathrm{Xe}, \mathrm{Si}, \mathrm{C}$, and $\mathrm{N}$, Geochim. Cosmochim. Acta, 53, 3273-3290, 1989.

T. Yoshida (e-mail: tyoshida@geo.titech.ac.jp), H. Emori, and K. Nakazawa 